

\title{
Photo- and Electroproduction of Eta Mesons*
}

\author{
G. Knöchlein, D. Drechsel, L. Tiator \\ Institut für Kernphysik, Johannes Gutenberg-Universität, D-55099 Mainz, Germany \\ E-Mail: knoechlein@vkpmzp.kph.uni-mainz.de
}

Eta photo- and electroproduction off the nucleon is investigated in an effective lagrangian approach that contains Born terms and both vector meson and nucleon resonance contributions. In particular, we review and develop the formalism for coincidence experiments with polarization degrees of freedom. The different response functions appearing in single and double polarization experiments have been studied. We will present calculations for structure functions and kinematical conditions that are most sensitive to details of the lagrangian, in particular with regard to contributions of nucleon resonances beyond the dominant $S_{11}(1535)$ resonance.

${ }^{*}$ This work has been supported by the Deutsche Forschungsgemeinschaft (SFB 201). 


\section{INTRODUCTION}

The advent of new electron accelerators and intense photon sources has given rise to a renewed interest in photo-and electroproduction of eta mesons. Previous attempts of the late 60's to study this process were severely limited by large error bars [1] 6], and also a few later measurements did not substantially improve the data basis [7] 8 ]. The recent data of the TAPS collaboration at MAMI have shown, however, that this situation is rapidly changing by use of modern accelerators and detection equipment [9]. The high-quality data for angular distributions and total cross sections for photon energies between threshold and $790 \mathrm{MeV}$ may be considered to be a qualitative break-through in the experimental field. Similarly, data at the higher energies up to $1150 \mathrm{MeV}$ will be provided soon by the Phoenics collaboration at ELSA 10. At present, the data basis for electroproduction of eta mesons is still very scarce. It is limited to a few older Bonn data [1] with large error bars and a more recent investigation of the Elan collaboration at ELSA at very small momentum transfer, $Q^{2}=0.056 \mathrm{GeV}^{2}$ [12].

Since eta production is strongly dominated by the resonance $S_{11}(1535)$, its coupling to other resonances is difficult to extract even from very precise angular distributions. Instead, polarization observables will have to play a major role in order to constrain the small resonance couplings. Such experiments have been proposed, e.g., at the laser backscattering facilities LEGS at Brookhaven and GRAAL at Grenoble. The experiments at GRAAL will cover the energy range up to $2 \mathrm{GeV}$ in the c.m. frame and provide a first look at $\eta^{\prime}$ production. Moreover, a series of experiments has been planned at CEBAF to study both $\eta$ and $\eta^{\prime}$ production by use of polarization degrees of freedom [13].

In contrast to the isovector pions which excite both $\Delta(T=3 / 2)$ as well as $N^{*}(T=1 / 2)$ resonances, the isoscalar eta meson can couple only to resonances with $T=1 / 2$. However, a fascinating property of the eta is its strong coupling to the $S_{11}(1535)$ and a very weak coupling to all other $N^{*}$ resonances. Since the $S_{11}(1535)$ has a $45-55 \%$ branching ratio for eta decay, the eta is the ideal probe to "tag" and investigate this resonance. In comparison the $S_{11}(1650)$ has only a $1 \%$ branching ratio for eta decay. This fact has been explained in the quark model by a strong mixing between these two resonances [14]. It is less obvious why none of the other resonances seems to couple strongly to the eta. In particular, the neighbouring dipole excitation $D_{13}(1520)$ has a branching ratio of the order of $10^{-3}$. The coupling of the Roper resonance $P_{11}(1440)$ to the eta meson is difficult to determine, because the resonance mass is below threshold. A previously reported strong coupling to the $P_{11}(1710)$ has been dropped in the last issue of the Particle Data Group [15], because the presented evidence is not convincing. Further resonances with branching ratios of the order of $1 \%$ or less are the states $D_{15}(1675), D_{13}(1700)$ and $P_{13}(1720)$ 15. 16]. All of these states appear as resonances in $\pi N$ phase shift analyses, but precisely the $S_{11}(1535)$ is not a good candidate for a resonance in the $\pi N$ system. The speed-plot of the $\pi N S$-wave phase shift shows a very untypical form for a resonance [17, 18, probably because of a strong cusp effect due to the coupling to the eta channel.

$S U(3)$ flavour symmetry relates the coupling constant between the eta and the nucleon to the pion-nucleon interaction,

$$
\frac{g_{\eta N N}^{2}}{4 \pi}=\frac{1}{3}\left(3-4 \frac{D}{D+F}\right)^{2} \frac{g_{\pi N N}^{2}}{4 \pi} .
$$

The resulting value ranges between 0.8 and 1.9 and depends strongly on the strength parameters F and D of the two types of octet meson-baryon couplings. A previous analysis has shown, however, that much smaller values of the order of 0.4 are necessary to fit the data for eta photoproduction [19]. In the same analysis, the forward-backward asymmetry of the angular distribution [9] gave rise to the interesting observation that pseudoscalar (PS) coupling is to be favoured over pseudovector (PV) coupling. This finding seems to contrast the derivation of low energy theorems (LET), which require PV coupling for reasons of chiral invariance. However, such theorems are probably irrelevant for eta production because of the following reasons. LET describes the dominant $S$-wave multipole $E_{0+}$ by a power series in the ratio of eta and nucleon mass, $m_{\eta} / m_{N}$. Obviously, this expansion is not expected to converge well. Even in the much more favourable case of pion photoproduction with $\mu=m_{\pi} / m_{N} \approx 1 / 7$, the low-energy limit of $E_{0+}$ is difficult to predict. As has been shown in detailed calculations within the framework of chiral perturbation theory [20], higher loop effects play a major role and even a calculation up to terms in $\mu^{3}$ does not seem to be sufficient. Second, the $S_{11}(1535)$ is close to threshold and dominates the cross section. In view of such a nearby singularity in the complex energy plane, a power series expansion in $m_{\eta}$ is not expected to work near eta threshold. Third, the physical $\eta$ and $\eta^{\prime}$ mesons are combinations of the corresponding particles in the $S U(3)$ octet and singlet with a mixing angle of about $-20^{\circ}$. In this way the $U_{A}(1)$ anomaly of the axial singlet current will also affect the eta meson, and the corresponding axial current will not be conserved, even in the Goldstone limit of massless particles.

As has been pointed out, the slight backward peaking of the angular distributions can be explained by PS coupling between eta and nucleon, while a calculation with PV coupling would lead to a forward peaked cross section. The difference between the two schemes is due to a sign change in the $P$-wave $M_{1-}$ multipole. This multipole would also be affected by a sizeable eta branch of the $N^{*}(1440)$ or Roper resonance. Though there are no indications of such an effect at present, a careful study of the smaller multipoles in eta photoproduction is certainly well motivated. 
Previous attempts to describe eta photoproduction have involved Breit-Wigner functions for the resonances [21] or relativistic lagrangians to describe resonant and nonresonant contributions [22. In a basically nonrelativistic approximation, coupled-channel calculations have been developed to treat the reactions $\pi N \rightarrow \pi N, \pi N \rightarrow \pi \pi N$ and $\pi N \rightarrow \eta N$ in a unitary way 23 25]. Unfortunately the corresponding $3 \times 3 S$-matrix of the hadronic sector is not sufficiently well determined by the existing data, which leaves a considerable model dependence. It is interesting to see that a generalized Lie model can describe the hadronic data perfectly well, both with and without an explicit resonance [26]. In the latter case the resonance-like structure of the data is built up by a cusp effect due to the strong $\pi-\eta$ coupling. Though the two models lead to completely different singularities in the complex energy plane, the present data are not sufficient to discard one or the other option.

In the following calculations we will follow the model of Bennhold and Tanabe [23], which has already been applied to eta photoproduction in the region of the $S_{11}(1535)$. We will extend the model to higher energies and include higher resonances in a phenomenological way. Moreover, we will study the potential of polarization degrees of freedom in both photo- and electroproduction. It is our main aim to select kinematical situations and polarization observables most favourable for identifying higher multipole contributions in the presence of the strong $S$-wave transition.

In Sect. 2 we will outline the formalism to describe photo- and electroproduction up to the most general polarization experiment. The ingredients of a phenomenological model for eta production will be discussed in Sect. 3. Our results for photo- and electroproduction observables will be presented in Sects. 4 and 5, respectively, followed by a brief conclusion in Sect. 6.

\section{FORMALISM AND POLARIZATION DEGREES OF FREEDOM}

To lowest order of the fine structure constant $\alpha=e^{2} / 4 \pi \approx 1 / 137$ the electron interacts with the currents of the hadronic system through the exchange of one virtual photon with well defined energy, $\omega=\varepsilon_{f}-\varepsilon_{i}$, and momentum transfer, $\vec{q}=\vec{k}_{f}-\vec{k}_{i}$. The Lorentz vectors $k_{i}=\left(\varepsilon_{i}, \vec{k}_{i}\right)$ and $k_{f}=\left(\varepsilon_{f}, \vec{k}_{f}\right)$ characterize the kinematics of the incident and outgoing electron. In contrast to a photoproduction experiment, where photon energy and momentum are related by $q^{2}=\omega^{2}-\vec{q}^{2}=0$, the virtual photon offers the possibility of an independent variation of photon energy and momentum, leading to information on the spatial structure of the hadronic system. As a consequence the kinematical situation in an electron scattering experiment (see also Fig. E) is determined by three independent kinematical variables, which are usually chosen to be the invariant energy $W$, the scattering angle in the c.m. system $\Theta$ and the momentum transfer $Q^{2}=-q^{2}$. In a photoproduction experiment only two kinematical variables are required to describe the kinematics uniquely. In this case the usual choice of variables is the scattering angle $\Theta$ and the photon energy in the laboratory system $\nu$.

The nucleon is characterized by the Lorentz vector $P_{i}=\left(E_{i}, \vec{P}_{i}\right)$ and $P_{f}=\left(E_{f}, \vec{P}_{f}\right)$ in the initial and final state, respectively, and the 4 -momentum of the eta meson is denoted by $k=\left(\omega_{\eta}, \vec{k}\right)$. Of course, the whole formalism presented here can be easily applied to other pseudoscalar meson electroproduction experiments, e.g. pion production. The threshold photon energy for eta production is

$$
\nu_{t h r}=\frac{2 m_{N} m_{\eta}+m_{\eta}^{2}+Q^{2}}{2 m_{N}} .
$$

Using the notation [27], the differential cross section for the electroproduction process can be written as

$$
\begin{aligned}
d \sigma= & \frac{\varepsilon_{i}}{k_{i}} \frac{m_{e}}{\varepsilon_{i}} \frac{m_{N}}{E_{i}} \frac{m_{e}}{\varepsilon_{f}} \frac{d^{3} k_{f}}{(2 \pi)^{3}} \frac{1}{2 \omega_{\eta}} \frac{d^{3} k}{(2 \pi)^{3}} \frac{m_{N}}{E_{f}} \frac{d^{3} P_{f}}{(2 \pi)^{3}} \\
& \times(2 \pi)^{4} \delta^{(4)}\left(P_{i}+q-k-P_{f}\right) \\
& \times\left|\left\langle P_{f}, k\left|J^{\mu}\right| P_{i}\right\rangle q^{-2}\left\langle k_{f}\left|j_{\mu}\right| k_{i}\right\rangle\right|^{2},
\end{aligned}
$$

where the phase space is evaluated in the laboratory frame, and $j^{\mu}$ and $J^{\mu}$ denote the electromagnetic currents of the electron and the hadronic system, respectively.

The most general lorentz- and gauge invariant current operator $J^{\mu}$ for the production of a pseudoscalar particle off a nucleon between nucleon Dirac spinors is given by the CGLN parametrization [28] of the transition current,

$$
\begin{aligned}
\vec{J}= & \frac{4 \pi W}{m}\left[i \tilde{\boldsymbol{\sigma}} F_{1}+(\boldsymbol{\sigma} \cdot \hat{\vec{k}})(\boldsymbol{\sigma} \times \hat{\vec{q}}) F_{2}+i \tilde{\vec{k}}(\boldsymbol{\sigma} \cdot \hat{\vec{q}}) F_{3}\right. \\
& \left.+i \tilde{\vec{k}}(\boldsymbol{\sigma} \cdot \hat{\vec{k}}) F_{4}+i \hat{\vec{q}}(\boldsymbol{\sigma} \cdot \hat{\vec{q}}) F_{5}+i \hat{\vec{q}}(\boldsymbol{\sigma} \cdot \hat{\vec{k}}) F_{6}\right], \\
\rho= & \frac{4 \pi W}{m}\left[i(\boldsymbol{\sigma} \cdot \hat{\vec{k}}) F_{7}+i(\boldsymbol{\sigma} \cdot \hat{\vec{q}}) F_{8}\right]=\frac{\vec{q} \cdot \vec{J}}{\omega}
\end{aligned}
$$


with all vectors expressed in the c.m. frame and $\tilde{\vec{\sigma}}=\vec{\sigma}-(\vec{\sigma} \cdot \hat{\vec{q}}) \hat{\vec{q}}$ etc. In this parametrization the first four terms represent the purely transverse component, $\vec{J}_{T}$, of the current $\vec{J}$, while the fifth and sixth term are the longitudinal part, $\vec{J}_{L}$. The longitudinal component of the current is related to the scalar density $\rho$ via current conservation, $q_{\mu} J^{\mu}=0$, and, as a consequence,

$$
|\vec{q}| F_{5}=\omega F_{8},|\vec{q}| F_{6}=\omega F_{7} .
$$

The CGLN amplitudes are complex functions of three independent kinematical variables, $F_{i}=F_{i}\left(W, \Theta, Q^{2}\right)$. The degree of transverse polarization of the virtual photon,

$$
\varepsilon=\left(1+\frac{2 \vec{q}^{2}}{Q^{2}} \tan ^{2} \frac{\Theta_{e}}{2}\right)^{-1}
$$

is invariant under collinear transformations, and $\vec{q}$ and $\Theta_{e}$ may be expressed in the lab or c.m. frame, while the longitudinal polarization,

$$
\varepsilon_{L}=\frac{Q^{2}}{\omega^{2}} \varepsilon,
$$

is frame-dependent. Using standard techniques (see e.g. [29] or [30]), the differential cross section for an electroproduction experiment is

$$
\frac{d \sigma}{d \Omega_{f} d \varepsilon_{f} d \Omega_{\eta}}=\Gamma \frac{d \sigma_{v}}{d \Omega_{\eta}}
$$

where the flux of the virtual photon field is given by

$$
\Gamma=\frac{\alpha}{2 \pi^{2}} \frac{\varepsilon_{f}}{\varepsilon_{i}} \frac{k_{\gamma}^{l a b}}{Q^{2}} \frac{1}{1-\varepsilon},
$$

and the virtual differential cross section is

$$
\begin{aligned}
\frac{d \sigma_{v}}{d \Omega_{\eta}}= & \frac{d \sigma_{T}}{d \Omega_{\eta}}+\varepsilon_{L} \frac{d \sigma_{L}}{d \Omega_{\eta}}+\left[2 \varepsilon_{L}(1+\varepsilon)\right]^{1 / 2} \frac{d \sigma_{T L}}{d \Omega_{\eta}} \cos \phi_{\eta} \\
& +\varepsilon \frac{d \sigma_{T T}}{d \Omega_{\eta}} \cos 2 \phi_{\eta}+h\left[2 \varepsilon_{L}(1-\varepsilon)\right]^{1 / 2} \frac{d \sigma_{T L^{\prime}}}{d \Omega_{\eta}} \sin \phi_{\eta} \\
& +h\left(1-\varepsilon^{2}\right)^{1 / 2} \frac{d \sigma_{T T^{\prime}}}{d \Omega_{\eta}} .
\end{aligned}
$$

In this expression $k_{\gamma}^{l a b}=\left(W^{2}-m_{i}^{2}\right) / 2 m_{i}$ denotes the "photon equivalent energy", the laboratory energy necessary for a real photon to excite a hadronic system with c.m. energy $W$. Note that all kinematical variables appearing in the virtual photon cross section $d \sigma_{v} / d \Omega_{\eta}$ have to be expressed in the c.m. frame. The individual contributions $d \sigma_{i} / d \Omega_{\eta}\left(i=T, L, T L, T T, T L^{\prime}, T T^{\prime}\right)$ are usually parametrized in terms of response functions which depend on the independent kinematical variables.

Experiments with three types of polarization can be performed in eta production: electron beam polarization, polarization of the target nucleon and polarization of the recoil nucleon. Target polarization will be described in the frame $\{x, y, z\}$ in Fig. $\mathbf{E}$, with the $z$-axis pointing into the direction of the photon momentum $\hat{\vec{q}}$, the $y$-axis perpendicular to the reaction plane, $\hat{\vec{y}}=\hat{\vec{q}} \times \hat{\vec{k}} / \sin \Theta_{\eta}$, and the $x$-axis given by $\hat{\vec{x}}=\hat{\vec{y}} \times \hat{\vec{z}}$. For recoil polarization we will use the frame $\left\{x^{\prime}, y^{\prime}, z^{\prime}\right\}$, with the $z^{\prime}$-axis defined by the momentum vector of the outgoing eta meson $\hat{\vec{k}}$, the $y^{\prime}$-axis as for target polarization and the $x^{\prime}$-axis given by $\hat{\vec{x}}^{\prime}=\hat{\vec{y}}^{\prime} \times \hat{\vec{z}}^{\prime}$. The most general expression for a coincidence experiment considering all three types of polarization is

$$
\begin{aligned}
\frac{d \sigma_{v}}{d \Omega_{\eta}}= & \frac{|\vec{k}|}{k_{\gamma}^{c m}} P_{\alpha} P_{\beta}\left\{R_{T}^{\beta \alpha}+\varepsilon_{L} R_{L}^{\beta \alpha}\right. \\
& +\left[2 \varepsilon_{L}(1+\varepsilon)\right]^{1 / 2}\left({ }^{c} R_{T L}^{\beta \alpha} \cos \phi_{\eta}+{ }^{s} R_{T L}^{\beta \alpha} \sin \phi_{\eta}\right) \\
& +\varepsilon\left({ }^{c} R_{T T}^{\beta \alpha} \cos 2 \phi_{\eta}+{ }^{s} R_{T T}^{\beta \alpha} \sin 2 \phi_{\eta}\right) \\
& +h\left[2 \varepsilon_{L}(1-\varepsilon)\right]^{1 / 2}\left({ }^{c} R_{T L^{\prime}}^{\beta \alpha} \cos \phi_{\eta}+{ }^{s} R_{T L^{\prime}}^{\beta \alpha} \sin \phi_{\eta}\right) \\
& \left.+h\left(1-\varepsilon^{2}\right)^{1 / 2} R_{T T^{\prime}}^{\beta \alpha}\right\}
\end{aligned}
$$


where $P_{\alpha}=(1, \vec{P})$ and $P_{\beta}=\left(1, \vec{P}^{\prime}\right)$. Here $\vec{P}=\left(P_{x}, P_{y}, P_{z}\right)$ denotes the target and $\vec{P}^{\prime}=\left(P_{x^{\prime}}, P_{y^{\prime}}, P_{z^{\prime}}\right)$ the recoil polarization vector. The zero components $P_{0}=1$ lead to contributions in the cross section which are present in the polarized as well as the unpolarized case. In an experiment without target and recoil polarization $\alpha=\beta=0$, so the only remaining contributions are $R_{i}^{00}$. The functions $R_{i}^{\beta \alpha}$ describe the response of the hadronic system in the process. Summation over Greek indices is implied. An additional superscript $s$ or $c$ on the left indicates a sine or cosine dependence of the respective contribution on the azimuthal angle. Some response functions vanish identically (see Table for a systematic overview ). The number of different response functions is further reduced by the equalities listed in App. A, and in the most general electroproduction experiment 36 polarization observables can be determined. The response functions $R_{i}^{\beta \alpha}$ are real or imaginary parts of bilinear forms of the CGLN amplitudes depending on the scattering angle $\Theta$.

TABLE I. Polarization observables in pseudoscalar meson electroproduction. A star denotes a response function which does not vanish but is identical to another response function via a relation in App. A.

\begin{tabular}{|c|c|c|c|c|c|c|c|c|c|c|c|c|c|c|c|c|}
\hline \multirow[b]{2}{*}{$\beta$} & \multirow[b]{2}{*}{-} & \multicolumn{3}{|c|}{ Target } & \multicolumn{3}{|c|}{ Recoil } & \multicolumn{9}{|c|}{ Target + Recoil } \\
\hline & & - & - & - & $x^{\prime}$ & $y^{\prime}$ & $z^{\prime}$ & $x^{\prime}$ & $x^{\prime}$ & $x^{\prime}$ & $y^{\prime}$ & $y^{\prime}$ & $y^{\prime}$ & $z^{\prime}$ & $z^{\prime}$ & $z^{\prime}$ \\
\hline$\alpha$ & - & $x$ & $y$ & $z$ & - & - & - & $x$ & $y$ & $z$ & $x$ & $y$ & $z$ & $x$ & $y$ & $z$ \\
\hline$T$ & $R_{T}^{00}$ & 0 & $R_{T}^{0 y}$ & 0 & 0 & $R_{T}^{y^{\prime} 0}$ & 0 & $R_{T}^{x^{\prime} x}$ & 0 & $R_{T}^{x^{\prime} z}$ & 0 & $*$ & 0 & $R_{T}^{z^{\prime} x}$ & 0 & $R_{T}^{z^{\prime} z}$ \\
\hline$L$ & $R_{L}$ & 0 & $R_{L}^{0 y}$ & 0 & 0 & $*$ & 0 & $R_{L}^{x^{\prime} x}$ & 0 & $R_{L}^{x^{\prime} z}$ & 0 & * & 0 & $*$ & 0 & * \\
\hline${ }^{c} T L$ & ${ }^{c} R_{T L}^{00}$ & 0 & ${ }^{c} R_{T L}^{0 y}$ & 0 & 0 & $*$ & 0 & ${ }^{c} R_{T L}^{x^{\prime} x}$ & 0 & $*$ & 0 & $*$ & 0 & ${ }^{c} R_{T L}^{z^{\prime} x}$ & 0 & * \\
\hline${ }^{s} T L$ & 0 & ${ }^{s} R_{T L}^{0 x}$ & 0 & ${ }^{s} R_{T L}^{0 z}$ & ${ }^{s} R_{T L}^{x^{\prime} 0}$ & 0 & ${ }^{s} R_{T L}^{z^{\prime} 0}$ & 0 & * & 0 & $*$ & 0 & $*$ & 0 & * & 0 \\
\hline${ }^{c} T T$ & ${ }^{c} R_{T T}^{00}$ & 0 & * & 0 & 0 & * & 0 & * & 0 & * & 0 & * & 0 & * & 0 & * \\
\hline${ }^{s} T T$ & 0 & ${ }^{s} R_{T T}^{0 x}$ & 0 & ${ }^{s} R_{T T}^{0 z}$ & ${ }^{s} R_{T T}^{x^{\prime} 0}$ & 0 & ${ }^{s} R_{T T}^{z^{\prime} 0}$ & 0 & $*$ & 0 & $*$ & 0 & $*$ & 0 & $*$ & 0 \\
\hline${ }^{c} T L^{\prime}$ & 0 & ${ }^{c} R_{T L^{\prime}}^{0 x}$ & 0 & ${ }^{c} R_{T L^{\prime}}^{0 z}$ & ${ }^{c} R_{T L^{\prime}}^{x^{\prime}}$ & 0 & ${ }^{c} R_{T L^{\prime}}^{z^{\prime}}$ & 0 & $*$ & 0 & $*$ & 0 & * & 0 & $*$ & 0 \\
\hline${ }^{s} T L^{\prime}$ & ${ }^{s} R_{T L^{\prime}}^{00}$ & 0 & ${ }^{s} R_{T L^{\prime}}^{0 y}$ & 0 & 0 & * & 0 & ${ }^{s} R_{T L^{\prime}}^{x^{\prime} x}$ & 0 & * & 0 & $*$ & 0 & ${ }^{s} R_{T L^{\prime}}^{z^{\prime} x}$ & 0 & * \\
\hline$T T^{\prime}$ & 0 & $R_{T T^{\prime}}^{0 x}$ & 0 & $R_{T T^{\prime}}^{0 z}$ & $R_{T T^{\prime}}^{x^{\prime} 0}$ & 0 & $R_{T T^{\prime}}^{z^{\prime} 0}$ & 0 & * & 0 & * & 0 & * & 0 & * & 0 \\
\hline
\end{tabular}

In photoproduction the longitudinal components vanish, and the relevant response functions will be divided by the transverse response function $R_{T}^{00}$ in order to obtain the polarization observables. The common descriptors of these observables (see also [31] and [32]) can be found in Table [I], the relation to the response functions in App. B.

TABLE II. Polarization observables in meson photoproduction. The entries in brackets denote polarization observables, which also appear elsewhere in the table.

\begin{tabular}{|c|c|c|c|c|c|c|c|c|c|c|c|}
\hline \multirow[t]{2}{*}{ Photon } & \multirow{3}{*}{$\begin{array}{l}- \\
- \\
\end{array}$} & \multicolumn{3}{|c|}{ Target } & \multicolumn{3}{|c|}{ Recoil } & \multicolumn{4}{|c|}{ Target + Recoil } \\
\hline & & - & - & - & $x^{\prime}$ & $y^{\prime}$ & $z^{\prime}$ & $x^{\prime}$ & $x^{\prime}$ & $z^{\prime}$ & $z^{\prime}$ \\
\hline & & $x$ & $y$ & $z$ & - & - & - & $x$ & $z$ & $x$ & $z$ \\
\hline transverse unpolarized $(T)$ & $\sigma_{0}$ & 0 & $T$ & 0 & 0 & $P$ & 0 & $T_{x^{\prime}}$ & $-L_{x^{\prime}}$ & $T_{z^{\prime}}$ & $L_{z^{\prime}}$ \\
\hline transverse (linear pol.) $(T T)$ & $-\Sigma$ & $H$ & $(-P)$ & $-G$ & $O_{x^{\prime}}$ & $(-T)$ & $O_{z^{\prime}}$ & $\left(-L_{z^{\prime}}\right)$ & $\left(T_{z^{\prime}}\right)$ & $\left(-L_{x^{\prime}}\right)$ & $\left(-T_{x^{\prime}}\right)$ \\
\hline transverse (circular pol.) $\left(T T^{\prime}\right)$ & 0 & $F$ & 0 & $-E$ & $-C_{x^{\prime}}$ & 0 & $-C_{z^{\prime}}$ & 0 & 0 & 0 & 0 \\
\hline
\end{tabular}


In contrast to electroproduction, there are no new polarization observables in photoproduction accessible by triple polarization experiments (beam + target + recoil polarization). As a consequence we may classify the differential cross sections by the three classes of double polarization experiments:

- polarized photons and polarized target

$$
\begin{aligned}
\frac{d \sigma}{d \Omega_{\eta}}= & \sigma_{0}\left\{1-P_{T} \Sigma \cos 2 \varphi\right. \\
& +P_{x}\left(-P_{T} H \sin 2 \varphi+P_{\odot} F\right) \\
& -P_{y}\left(-T+P_{T} P \cos 2 \varphi\right) \\
& \left.-P_{z}\left(-P_{T} G \sin 2 \varphi+P_{\odot} E\right)\right\}
\end{aligned}
$$

- polarized photons and recoil polarization

$$
\begin{aligned}
\frac{d \sigma}{d \Omega_{\eta}}= & \sigma_{0}\left\{1-P_{T} \Sigma \cos 2 \varphi\right. \\
& +P_{x^{\prime}}\left(-P_{T} O_{x^{\prime}} \sin 2 \varphi-P_{\odot} C_{x^{\prime}}\right) \\
& -P_{y^{\prime}}\left(-P+P_{T} T \cos 2 \varphi\right) \\
& \left.-P_{z^{\prime}}\left(P_{T} O_{z^{\prime}} \sin 2 \varphi+P_{\odot} C_{z^{\prime}}\right)\right\},
\end{aligned}
$$

- polarized target and recoil polarization

$$
\begin{aligned}
\frac{d \sigma}{d \Omega_{\eta}}= & \sigma_{0}\left\{1+P_{y^{\prime}} P+P_{x}\left(P_{x^{\prime}} T_{x^{\prime}}+P_{z^{\prime}} T_{z^{\prime}}\right)\right. \\
& \left.+P_{y}\left(T+P_{y^{\prime}} \Sigma\right)-P_{z}\left(P_{x^{\prime}} L_{x^{\prime}}-P_{z^{\prime}} L_{z^{\prime}}\right)\right\} .
\end{aligned}
$$

In these equations $\sigma_{0}$ denotes the unpolarized differential cross section, the transverse degree of photon polarization is denoted by $P_{T}, P_{\odot}$ is the right-handed circular photon polarization and $\varphi$ the angle between photon polarization vector and reaction plane.

In conclusion there are 16 different polarization observables for real photon experiments. In electroproduction there are four additional observables for the exchange of longitudinal photons and sixteen observables due to longitudinaltransverse interference. However, as has become clear from the general form of the transition current $J^{\mu}$, there are only six independent complex amplitudes uniquely describing the electroproduction process. This corresponds to six absolute values and five relative phases between the CGLN amplitudes, i.e. there are only eleven independent quantities which completely and uniquely determine the transition current $J^{\mu}$.

The aim of a so-called "complete experiment" is the determination of the current $J^{\mu}$ for the process under investigation in a given kinematics. In order to carry through such a complete experiment, it should suffice to determine eleven independent response functions. The ambitious program of a complete experiment was formulated by Barker et al. in the sixties for the photoproduction case [31]. Although it would be interesting to pursue such a project for eta production, we will concentrate in the following on the more physical aspects of this process. In particular our theoretical investigations will supply information on an adequate selection of response functions and observables. In the case of eta production it will be of special interest to study response functions which give clear information of the eta meson coupling to higher resonances.

Another useful parametrization of the hadronic current $\vec{J}$ is based on a decomposition into spherical components $J_{ \pm, 0}$, with $J_{ \pm}=\mp\left(J_{x} \pm i J_{y}\right) / \sqrt{2}$ and $J_{0}=J_{z}$. Since the components of the current operator $\vec{J}$ are operators in two-dimensional Pauli spinor space, we can represent them by $2 \times 2$ matrices in terms of the helicity amplitudes $H_{i}$ :

$$
\begin{aligned}
J_{+} & =\left(\begin{array}{ll}
H_{1} & H_{2} \\
H_{3} & H_{4}
\end{array}\right), J_{-}=\left(\begin{array}{cc}
H_{4} & -H_{3} \\
-H_{2} & H_{1}
\end{array}\right), \\
J_{0} & =\left(\begin{array}{cc}
H_{5} & H_{6} \\
H_{6} & -H_{5}
\end{array}\right) .
\end{aligned}
$$

The response functions are expressed by these helicity amplitudes in App. D. It is straightforward to calculate the relation between CGLN and helicity amplitudes by comparing the matrix elements of the current operator (4) with the definitions (16) transformed into the Cartesian basis. The resulting transformations are 


$$
\begin{aligned}
H_{1} & =\frac{-1}{\sqrt{2}} \sin \Theta\left(F_{3}+F_{4} \cos \Theta\right), \\
H_{2} & =\frac{-1}{\sqrt{2}}\left(2 F_{1}-2 F_{2} \cos \Theta+F_{4} \sin ^{2} \Theta\right), \\
H_{3} & =\frac{-1}{\sqrt{2}} F_{4} \sin ^{2} \Theta, \\
H_{4} & =\frac{1}{\sqrt{2}} \sin \Theta\left(2 F_{2}+F_{3}+F_{4} \cos \Theta\right), \\
H_{5} & =F_{5}+F_{6} \cos \Theta, \\
H_{6} & =F_{6} \sin \Theta .
\end{aligned}
$$

Electroproduction data are usually analyzed in terms of multipoles $E_{l \pm}, M_{l \pm}$ and $L_{l \pm}$ characterizing the excitation mechanism (electric $(E)$, magnetic $(M)$ or longitudinal $(L))$ and the orbital $(l)$ and total angular momentum, which is given by $j=l \pm \frac{1}{2}$. The relation between these meson production multipoles and the type of electromagnetic multipole radiation $E L, M L, C L$ responsible for resonance excitation can be read off from Table IIII. The connection with the CGLN amplitudes can be established via a multipole series in terms of derivatives of the Legendre polynomials $P_{l}$ [33,

$$
\begin{aligned}
F_{1}= & \sum_{l \geq 0}\left\{\left(M_{l+}+E_{l+}\right) P^{\prime}{ }_{l+1}\right. \\
& \left.+\left[(l+1) M_{l-}+E_{l-}\right] P^{\prime}{ }_{l-1}\right\}, \\
F_{2}= & \sum_{l \geq 1}\left[(l+1) M_{l+}+l M_{l-}\right] P_{l}^{\prime}, \\
F_{3}= & \sum_{l \geq 1}\left[\left(E_{l+}-M_{l+}\right) P^{\prime \prime}{ }_{l+1}\right. \\
& \left.+\left(E_{l-}+M_{l-}\right) P^{\prime \prime}{ }_{l-1}\right], \\
F_{4}= & \sum_{l \geq 2}\left(M_{l+}-E_{l+}-M_{l-}-E_{l-}\right) P^{\prime \prime}{ }_{l}, \\
F_{5}= & \sum_{l \geq 0}\left[(l+1) L_{l+} P^{\prime}{ }_{l+1}-l L_{l-} P^{\prime}{ }_{l-1}\right], \\
F_{6}= & \sum_{l \geq 1}\left[l L_{l-}-(l+1) L_{l+}\right] P^{\prime}{ }_{l} .
\end{aligned}
$$

The inversion of this set of equations is very useful for constructing phenomenological models:

$$
\begin{aligned}
E_{l+}= & \int_{-1}^{1} d x\left[\frac{1}{2(l+1)} P_{l} F_{1}-\frac{1}{2(l+1)} P_{l+1} F_{2}\right. \\
& +\frac{1}{2(l+1)} \frac{l}{2 l+1}\left(P_{l-1}-P_{l+1}\right) F_{3} \\
& \left.+\frac{1}{2(2 l+3)}\left(P_{l}-P_{l+2}\right) F_{4}\right], \\
E_{l-}= & \int_{-1}^{1} d x\left[\frac{1}{2 l} P_{l} F_{1}-\frac{1}{2 l} P_{l-1} F_{2}\right. \\
& +\frac{l+1}{2 l(2 l+1)}\left(P_{l+1}-P_{l-1}\right) F_{3} \\
& \left.+\frac{1}{2(2 l-1)}\left(P_{l}-P_{l-2}\right) F_{4}\right], \\
M_{l+}= & \int_{-1}^{1} d x\left[\frac{1}{2(l+1)} P_{l} F_{1}-\frac{1}{2(l+1)} P_{l+1} F_{2}\right. \\
& \left.+\frac{1}{2(l+1)(2 l+1)}\left(P_{l+1}-P_{l-1}\right) F_{3}\right],
\end{aligned}
$$




$$
\begin{aligned}
M_{l-}= & \int_{-1}^{1} d x\left[-\frac{1}{2 l} P_{l} F_{1}+\frac{1}{2 l} P_{l-1} F_{2}\right. \\
& \left.+\frac{1}{2 l(2 l+1)}\left(P_{l-1}-P_{l+1}\right) F_{3}\right], \\
L_{l+}= & \int_{-1}^{1} d x \frac{1}{2(l+1)}\left[P_{l} F_{5}+P_{l+1} F_{6}\right], \\
L_{l-}= & \int_{-1}^{1} d x \frac{1}{2 l}\left[P_{l} F_{5}+P_{l+1} F_{6}\right] .
\end{aligned}
$$

The longitudinal multipole amplitudes $L_{l \pm}$ are related to the scalar amplitudes $S_{l \pm}$, which originate from the time-like component of the transition current (4), by current conservation,

$$
\omega S_{l \pm}=|\vec{q}| L_{l \pm} .
$$

Furthermore it is convenient to reconstruct the multipoles from amplitudes $A_{l \pm}, B_{l \pm}$ and $C_{l \pm}$, which are closely related to the electromagnetic resonance couplings $A_{1 / 2}^{N}, A_{3 / 2}^{N}$ and $C_{1 / 2}^{N}$ to be discussed in Sect. III:

$$
\begin{aligned}
E_{l+} & =\frac{1}{l+1} A_{l+}+\frac{l}{2(l+1)} B_{l+}, \\
E_{l-} & =-\frac{1}{l} A_{l-}+\frac{l+1}{2 l} B_{l-}, \\
M_{l+} & =\frac{1}{l+1} A_{l+}-\frac{l+2}{2(l+1)} B_{l+}, \\
M_{l-} & =\frac{1}{l} A_{l-}+\frac{l-1}{2 l} B_{l-}, \\
S_{l+} & =\frac{1}{l+1} C_{l+}, \\
S_{l-} & =-\frac{1}{l} C_{l-} .
\end{aligned}
$$

\section{A MODEL FOR ETA ELECTROPRODUCTION}

The dominant process for eta photoproduction is given by nucleon isobar excitation. In addition, background contributions from Born terms and $t$-channel vector meson exchange will be considered.

The resonance contributions in the direct channel are parametrized in the usual manner (see e.g. [34] or [35]) by means of Breit-Wigner helicity amplitudes $A_{l \pm}, B_{l \pm}$ and $C_{l \pm}$ in the relevant partial wave. These amplitudes are related to the multipole amplitudes through relations (36) to (41). In the construction of our model we will use the following definitions for these amplitudes:

$$
\begin{aligned}
& A_{l \pm}= \pm K A_{1 / 2}^{N}, \\
& B_{l \pm}=\mp K \sqrt{\frac{4}{l(l+2)} A_{3 / 2}^{N},} \\
& C_{l \pm}= \pm K C_{1 / 2}^{N} .
\end{aligned}
$$

The factor $K$ [36] describes the propagation and decay of an $N^{*}$ resonance and consists of a Breit-Wigner term and a phase space factor for the partial wave,

$$
K=\sqrt{\frac{k_{\gamma}^{c m}}{|\vec{k}|} \frac{m}{W} \frac{\Gamma_{\eta}}{\pi(2 J+1)}} \frac{M^{*}}{M^{*^{2}}-W^{2}-i W \Gamma} .
$$

The electromagnetic excitation of a resonance off the nucleon $N(N \in\{p, n\})$ is described by the electromagnetic helicity amplitudes $A_{1 / 2}^{N}, A_{3 / 2}^{N}$ and $C_{1 / 2}^{N}$ (see e.g. [37] or [38]), which consist of a standard kinematical factor $R=$ 
$\sqrt{2 \pi \alpha / k_{\gamma}^{c m}}$ and the matrix elements of spherical components $J_{\lambda}^{[j]^{i n t}}$ of the electromagnetic current operator at the photon-baryon vertex:

$$
\begin{aligned}
& A_{1 / 2}^{N}=R\left\langle N^{*}, J_{z}=\frac{1}{2}\left|J_{1}^{[1]^{i n t}}\right| N, J_{z}=-\frac{1}{2}\right\rangle, \\
& A_{3 / 2}^{N}=R\left\langle N^{*}, J_{z}=\frac{3}{2}\left|J_{1}^{[1]^{i n t}}\right| N, J_{z}=\frac{1}{2}\right\rangle, \\
& C_{1 / 2}^{N}=R\left\langle N^{*}, J_{z}=\frac{1}{2}\left|J_{0}^{[0]^{i n t}}\right| N, J_{z}=\frac{1}{2}\right\rangle .
\end{aligned}
$$

The simplest choice for deriving these matrix elements is the non-relativistic quark model, which we will use in our standard calculations. Within the framework of this model the current operator is given by

$$
\begin{aligned}
& \vec{J}(\vec{q})=\frac{1}{2 m_{q}} \sum_{j=1}^{3}\left(e_{j}\left\{\vec{p}_{j}, e^{i \vec{q} \cdot \vec{x}_{j}}\right\}-i \mu_{j} \vec{q} \times \vec{\sigma}_{j} e^{i \vec{q} \cdot \vec{x}_{j}}\right), \\
& \rho(\vec{q})=\sum_{j=1}^{3} e_{j} e^{i \vec{q} \cdot \vec{x}_{j}}
\end{aligned}
$$

The charge of a constituent quark $e_{j}$ is given in units of the electric charge $e$, and the constituent quark magnetic moment is given as $\mu_{j}=e_{j}$, because we treat the constituent quark as Dirac particles. For the wave functions of the baryonic states relevant in eta production we apply standard harmonic oscillator wavefunctions with configuration mixing in the $S_{11}(1535)$ and $D_{13}(1520)$ states according to [14]. The $u$ - and $d$-quark masses are taken to be $m_{q}=$ $350 \mathrm{MeV}$ [14], and for the oscillator spring constant we use the value of $300 \mathrm{MeV}$ [14]. The matrix elements are evaluated in the equal velocity frame. We will compare the electroproduction results obtained in this model (to be called M1 in the following) with the relativized constituent quark model (M2) by Warns et al. [38], the relativistic light cone calculation (M3) by Konen and Weber [39] and the light-front approach (M4) by Capstick and Keister [35]. Figs. $\mathrm{E}, \mathrm{E}$ and $\mathrm{E}$ present the matrix elements $A_{1 / 2}^{N}, A_{3 / 2}^{N}$ and $C_{1 / 2}^{N}$ for the resonances $S_{11}, D_{13}$ and $P_{11}$ as a function of the momentum transfer $Q^{2}$.

In (45) the branching fraction $\Gamma_{\eta} / \Gamma$ for resonance decay into nucleon and eta meson determines the strength of the coupling of the eta meson to the nucleon. In our model we use the branching fractions listed in Table III. For all hadronic widths necessary in the description of the resonance contributions we introduce the energy dependence suggested in [40] and [41], which results in the correct threshold behaviour of the multipole amplitudes with respect to the momentum of the outgoing meson $\vec{k}[42]$,

$$
E_{l \pm} \sim|\vec{k}|^{l}, \quad M_{l \pm} \sim|\vec{k}|^{l}, \quad L_{l \pm} \sim|\vec{k}|^{l} .
$$

The energy dependent total width of a resonance $\Gamma(W)$ is given by the sum over the partial widths of all possible decay channels,

$$
\Gamma(W)=\sum_{r} \Gamma_{r}(W)
$$

We construct the energy dependence of $\Gamma_{r}$ according to the $\pi N$ scattering analysis in [40]. The experimental data available on branching fractions usually refer to the branching fraction at the resonance energy. The energy dependence is introduced by the function $\rho_{j}$, which takes into account the penetration of the angular momentum barrier for total angular momentum $j$,

$$
\Gamma_{r}(W)=\Gamma_{r}\left(M^{*}\right) \frac{\rho_{j}(W)}{\rho_{j}\left(M^{*}\right)} .
$$

The functions $\rho_{j}$ are constructed from the Blatt-Weisskopf functions $B_{l}$ 43],

$$
\rho_{j}\left(M^{*}\right)=\frac{|\vec{k}|}{W} B_{l}^{2}(|\vec{k}| R) .
$$

The momentum $\vec{k}$ is the momentum of the outgoing meson in the c.m. frame. The effective interaction radius $R$ will be fixed at $1.0 \mathrm{fm}$. For the resonances under consideration we need the Blatt-Weisskopf functions for $l=0,1,2$, 


$$
\begin{aligned}
& B_{0}(x)=1 \\
& B_{1}(x)=\frac{x}{\sqrt{1+x^{2}}}, \\
& B_{2}(x)=\frac{x^{2}}{\sqrt{x^{4}+3 x^{2}+9}} .
\end{aligned}
$$

If one of the particles in the final state is unstable and decays into two particles with $m_{1}$ and $m_{2}$, while the stable particle in the final state has mass $m_{3}$, we calculate $\rho_{j}(W)$ by integration over the possible energy range for the decay products,

$$
\rho_{j}(W)=\int_{m_{1}+m_{2}}^{W-m_{3}} \sigma(\mathcal{M}) \frac{|\vec{k}|}{W} B_{l}^{2}(|\vec{k}| R) d \mathcal{M}
$$

where $\sigma(\mathcal{M})$ is a Breit-Wigner distribution,

$$
\sigma(\mathcal{M})=\frac{1}{2 \pi} \frac{\Gamma_{0}}{\left(\mathcal{M}-M_{0}\right)^{2}+\left(\Gamma_{0} / 2\right)^{2}} .
$$

In this parametrization $M_{0}$ is the mass of the unstable particle and $\Gamma_{0}$ its partial width. In the construction of the hadronic widths we essentially use resonance parameters obtained from the $\pi N$-scattering analysis [40]. We list the parameter set for the resonances relevant for our model in Table III.

TABLE III. Resonance parameters in our model. The symbol $\varepsilon$ represents an isoscalar two-pion-state with mass and width of $800 \mathrm{MeV}$ for the parametrization of uncorrelated two-pion production.

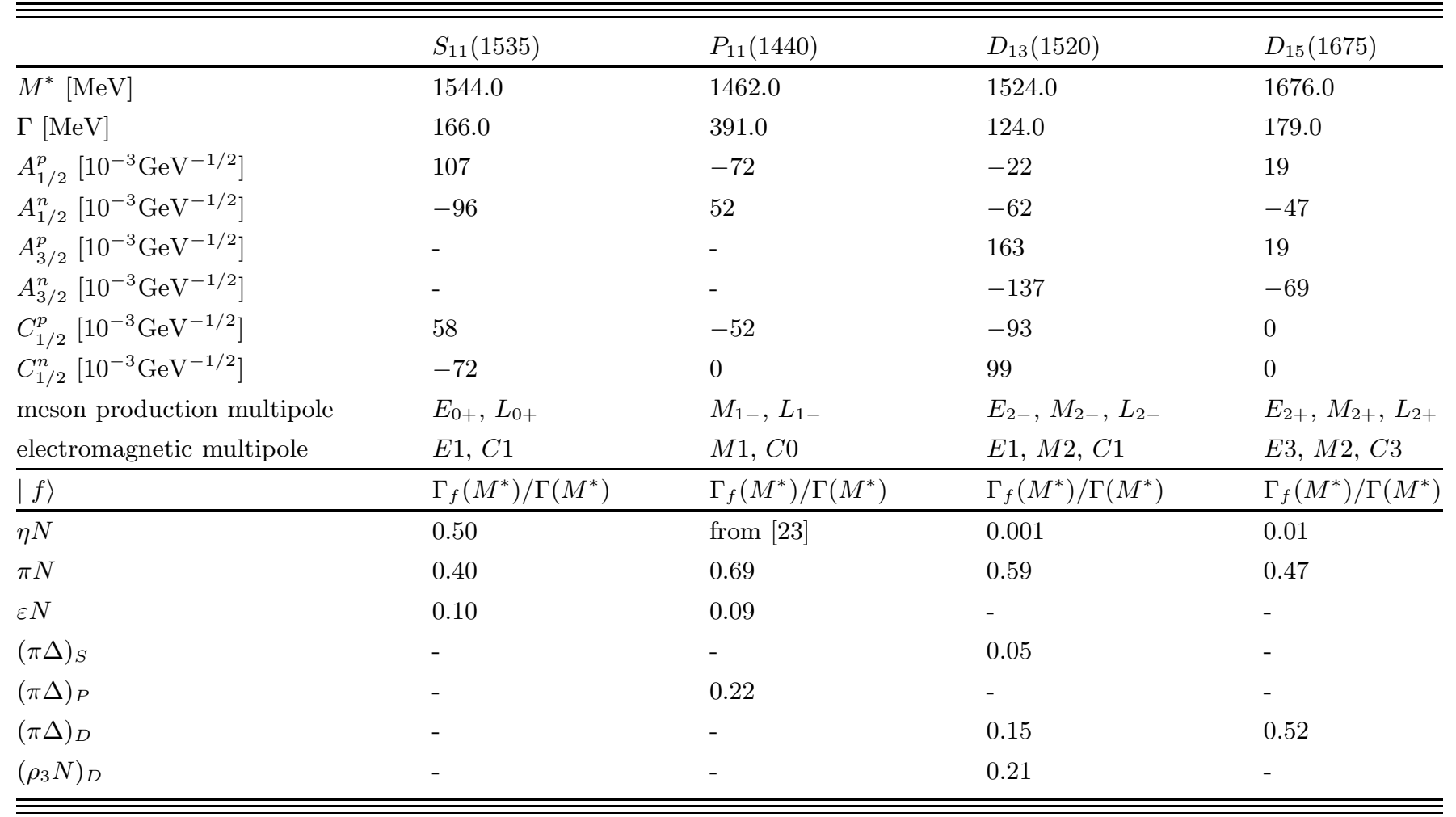


Unfortunately, the branching fraction $\Gamma_{\eta} / \Gamma$ of the Roper resonance cannot be treated within this parametrization, because the resonance energy is located below threshold. In this case we use the parametrization of the dynamical model by Bennhold and Tanabe [23] for the energy dependence of the partial width $\Gamma_{\eta}$.

For the evaluation of the Feynman diagrams contributing to the non-resonant background we use effective interaction lagrangian densities. The Born diagrams are calculated with the lagrangians

$$
\begin{aligned}
\mathcal{L}_{\eta N N}^{P S}= & -i g_{\eta N N} \bar{\psi} \gamma_{5} \psi \Phi_{\eta}, \\
\mathcal{L}_{\gamma N N}= & -e \bar{\psi}\left[\frac{1+\tau_{0}}{2} \gamma_{\mu} A^{\mu}\right. \\
& \left.-\left(\frac{\kappa_{p}+\kappa_{n}}{2}+\frac{\kappa_{p}-\kappa_{n}}{2} \tau_{0}\right) \frac{\sigma_{\mu \nu}}{2 m} \partial^{\nu} A^{\mu}\right] \psi,
\end{aligned}
$$

where $e=|e|>0$. The symbol $\kappa_{N}$ denotes the anomalous magnetic moment of the nucleon $\left(\kappa_{p}=1.79, \kappa_{n}=-1.91\right)$ and $g_{\eta N N}$ is the eta-nucleon coupling constant. We have chosen the pseudoscalar eta-nucleon coupling, because it fits the data for angular distributions above threshold [9, 12 much better than the alternative pseudovector coupling, which has been demonstrated in [19]. In this reference the value of the coupling constant was found to be $g_{\eta N N}^{2} / 4 \pi=0.4$, which is quite consistent with other approaches 44,45. In the electroproduction case we introduce the usual Dirac and Pauli form factors, $F_{1}\left(Q^{2}\right)$ and $F_{2}\left(Q^{2}\right)$, for the Dirac and Pauli currents, respectively. The connection with the standard dipole fit $F\left(Q^{2}\right)=\left(1+Q^{2} / 0.71 \mathrm{GeV}^{2}\right)^{-2}$ for the Sachs form factors is established by

$$
\begin{aligned}
& F_{1}^{p}=\frac{1+\tau\left(1+\kappa_{p}\right)}{1+\tau} F, \\
& F_{2}^{p}=\frac{1}{1+\tau} F, \\
& F_{1}^{n}=\frac{\tau \kappa_{n}}{1+\tau}\left(1-\frac{1}{1+4 \tau}\right) F, \\
& F_{2}^{n}=\frac{1}{1+\tau}\left(1-\frac{\tau}{1+4 \tau}\right) F,
\end{aligned}
$$

with $\tau=Q^{2} / 4 m^{2}$.

The effective lagrangians for the vector meson exchange vertices are given by

$$
\begin{aligned}
\mathcal{L}_{\gamma \eta V} & =\frac{g_{\gamma \eta V}}{m_{\eta}} \varepsilon_{\mu \nu \rho \sigma} \partial^{\mu} A^{\nu} \Phi_{\eta} \partial^{\rho} V^{\sigma}, \\
\mathcal{L}_{V N N} & =\bar{\psi}\left(g_{V_{1}} \gamma_{\mu}+\frac{g_{V_{2}}}{2 m} \sigma_{\mu \nu} \partial^{\nu}\right) V^{\mu} \psi .
\end{aligned}
$$

The parameters for the $\rho$ and $\omega$ mesons are listed in Table IV.

TABLE IV. Parameters for the vector mesons.

\begin{tabular}{llllll}
\hline \hline$V$ & $m_{V}[\mathrm{MeV}]$ & $g_{V_{1}}^{2} / 4 \pi$ & $g_{V_{2}} / g_{V_{1}}$ & $\Lambda_{V}[\mathrm{MeV}]$ & $\lambda_{V}$ \\
\hline$\omega$ & 782.6 & 23 & 0 & 1400 & 0.192 \\
$\rho$ & 769.0 & 0.5 & 6.1 & 1800 & 0.89 \\
\hline \hline
\end{tabular}


The electromagnetic couplings of the vector mesons $g_{\gamma \eta V}=e \lambda_{V} F_{V}\left(\vec{k}_{V}^{2}\right)$ are determined from the radiative widths. Following [46] the form factor $F_{V}$ is supposed to have the usual dipole behaviour. The hadronic couplings $g_{V_{1}}$ and $g_{V_{2}}$ are taken from a nuclear potential model [47] with the hadronic dipole form factor

$$
F_{V}^{h a d}\left(\vec{k}_{V}^{2}\right)=\frac{\left(\Lambda_{V}^{2}-m_{V}^{2}\right)^{2}}{\left(\Lambda_{V}^{2}+\vec{k}_{V}^{2}\right)^{2}}
$$

At tree level these contributions are real, they have been added to the real part of the resonance contribution. In general this procedure violates unitarity of the $S$-matrix. However, the unitarity corrections for most of the multipoles are small or even negligible in the threshold region, because the background contributions of most multipoles in eta production are small compared to the resonance contributions. Unfortunately, in eta production there is no simple constraint to the phase of the multipoles as in pion production, because the Watson theorem 448] is only valid in the elastic regime, whereas in eta production there are at least three hadronic reaction channels, $|N \pi\rangle,|N \eta\rangle$, and $|N \pi \pi\rangle$. In particular a consistent treatment of the state $|N \pi \pi\rangle$ is difficult, because it contains both uncorrelated two pion production and resonance mechanisms.

\section{RESULTS FOR PHOTOPRODUCTION}

The starting point of our model will be the latest TAPS [9] and Bonn [12] data on the total cross section for photoproduction of eta mesons on the proton 1 . A Breit-Wigner fit to these data,

$$
\sigma_{t o t}=\frac{|\vec{k}|}{|\vec{q}|} \frac{D M^{* 2} \Gamma(W)^{2}}{\left(M^{* 2}-W^{2}\right)^{2}+M^{* 2} \Gamma^{2}(W)},
$$

with a momentum dependent hadronic width,

$$
\Gamma(W)=\Gamma\left(M^{*}\right)\left(0.50 \frac{|\vec{k}|}{\left|\vec{k}^{*}\right|}+0.40 \frac{\left|\vec{k}_{\pi}\right|}{\left|\vec{k}_{\pi}^{*}\right|}+0.10\right),
$$

results in the following values for the three fit parameters: $M^{*}=1544.0 \mathrm{MeV}, \Gamma\left(M^{*}\right)=166.0 \mathrm{MeV}, D=39.0 \mu \mathrm{b}$. Note that we use the branching fractions $\Gamma_{\eta}\left(M^{*}\right) / \Gamma\left(M^{*}\right)=0.50, \quad \Gamma_{\pi}\left(M^{*}\right) / \Gamma\left(M^{*}\right)=0.40$ and $\Gamma_{\pi \pi}\left(M^{*}\right) / \Gamma\left(M^{*}\right)=$ 0.10 as input. At this point we have assumed, that the total cross section in this energy region is completely dominated by the $S_{11}(1535)$, which is justified by the small background, $P_{11}$, and $D_{13}$ contributions (see Fig. E). These additional contributions modify the shape of the total cross section in the $S_{11}(1535)$ region only slightly. Hence the electromagnetic helicity coupling $A_{1 / 2}^{p}$ of the $S_{11}(1535)$ at resonance energy $W=M^{*}$ is given by

$$
\left|A_{1 / 2}^{p}\right|=\sqrt{\frac{|\vec{k}|}{k_{\gamma}^{c m}} \frac{M^{*}}{m} \frac{2 D}{\Gamma_{\eta}\left(M^{*}\right)}} \frac{\Gamma\left(M^{*}\right)}{2} .
$$

The result of $107 \times 10^{-3} \mathrm{GeV}^{-1 / 2}$ is significantly larger than the standard value of $(68 \pm 10) \times 10^{-3} \mathrm{GeV}^{-1 / 2}$ in $[15$, but seems to be consistent with the value of $(95 \pm 11) \times 10^{-3} \mathrm{GeV}^{-1 / 2}$ from the analysis of eta production data [22] The TAPS analysis [9] yields values between 110 and $140 \times 10^{-3} \mathrm{GeV}^{-1 / 2}$ depending on the branching ratios of the $S_{11}$. Towards higher energies, the background contributions become more important, and our standard calculation is still consistent with eta production data from previous experiments [3, 4] within the large error bars. The latest preliminary data from Bonn [10], however, are in good agreement with our standard calculation in the energy range up to $W=1800 \mathrm{MeV}$.

The TAPS analysis of eta production data for total cross sections on the deuteron suggests that the elementary cross section on the neutron is about $80 \%$ of the proton value at the resonance peak [49], which corresponds to an electromagnetic helicity coupling of $A_{1 / 2}^{n}=-96 \times 10^{-3} \mathrm{GeV}^{-1 / 2}$ for the $S_{11}(1535)$ under the assumption of resonance

\footnotetext{
${ }^{1}$ The Bonn data [12] are electroproduction data at very low momentum transfer, $Q^{2}=0.056 \mathrm{GeV}^{2}$. Comparison with Fig. E justifies our treatment of these data as photoproduction data.
} 
dominance and is again significantly larger than the standard value 16 of $A_{1 / 2}^{n}=(-59 \pm 22) \times 10^{-3} \mathrm{GeV}^{-1 / 2}$. The $S_{11}$ dominance assumption is also supported by the rather flat angular distribution of the unpolarized differential cross section on the proton measured by the TAPS collaboration [9] (see Fig. E). Our standard calculation with background and resonance contributions of $S_{11}(1535), D_{13}(1520), D_{15}(1675)$ as well as $P_{11}(1440)$ is consistent with the data except for large angles. Omission of the Roper resonance results in an over-estimation of the differential cross section at small angles, whereas omission of the $D_{13}(1520)$ overestimates the differential cross section at medium and large angles. As the branching fraction of the $D_{13}(1520)$ resonance into the eta meson is not known precisely, we have also investigated a three- and tenfold increase in $\Gamma_{\eta}$. While the threefold increase in $\Gamma_{\eta}$ seems to improve the description of the data slightly, the tenfold increase is definitely not consistent with experiment. However, it has to be mentioned that the strength of a resonance contribution in a given multipole is determined by the factor $A_{\lambda}^{N} \sqrt{\Gamma_{\eta}} / \Gamma$, where $\lambda$ is the helicity of the intermediate resonance state. Therefore the uncertainties in the photocouplings $A_{\lambda}^{N}$ do also enter the strength of the respective multipole. Fig. 目 presents the unpolarized differential eta photoproduction cross sections off the proton and the neutron as a function of excitation energy $\nu$ and scattering angle $\Theta_{\eta}$. The calculations were performed in our standard parameter set, the cross section on the neutron was normalized to $80 \%$ of the proton value at resonance maximum. It is remarkable that the cross section for the neutron exposes a slight minimum at $90^{\circ}$, whereas the proton cross section has a slight maximum in this range of $\Theta_{\eta}$. This observation is compatible with the analysis in [19]. Having fixed the parameters of our model by unpolarized cross section data, we now proceed to study the polarization observables for single and double polarization experiments with beam and target polarization. All of these observables are accessible at the laser-backscattering facility GRAAL, where an extensive experimental program for eta photoproduction is already under way. Analysing the angular distributions of the observables in our model, it turns out that the most interesting effects can be seen at a scattering angle of

$90^{\circ}$. For this reason we choose this angle for the presentation of the excitation functions in Fig. 囵. The shape of the differential cross section does not expose any significant dependence on the $D_{13}$ or $P_{11}$ resonance. However, the non-resonant background destructively interferes with the $S_{11}$ contribution towards higher photon energies, which leads to a slight reduction of the total cross section. The photon asymmetry $\Sigma$ shows a very characteristic dependence on the contribution of the $D_{13}(1520)$ resonance due to interference of the $E_{2-}$ and $M_{2-}$ multipoles with the $E_{0+}$ multipole in ${ }^{c} R_{T T}^{00}$. Although the $D_{13}$ resonance has only a very small branching ratio into the eta meson, its absence would lead to an almost vanishing photon asymmetry within a wide range of excitation energies. Switching off the non-resonant background in the target or recoil asymmetry results in a value for these observables which is consistent with zero. In the threshold region the influence of the Roper resonance on the recoil asymmetry might also be visible in a precision experiment. Inspecting the double polarization observables for beam and target polarization, it becomes evident that the observable $-H$ behaves similar to the recoil asymmetry $P$ in the threshold region. Background and $D_{13}$ effects are also visible in the $G$ observable above threshold. The $E$ observable, however, is consistent with unity above threshold in each of the scenarios investigated. Since there are no additional spectacular effects in the double polarization observables for beam and recoil respectively target and recoil experiments, we will not present our results for these observables.

\section{RESULTS FOR ELECTROPRODUCTION}

At the electron scattering facilities ELSA and CEBAF (as well as at MAMI at low $Q^{2}$ in the threshold region) eta electroproduction on the proton can be studied with very high precision. As the available data are very scarce and have large error bars, the new experiments could tremendously extend our knowledge on this process. To our knowledge there has been no theoretical investigation of the eta electroproduction process in the literature before. Therefore, we will present some observables relevant for future experiments. Comparing Tables I and II, it becomes obvious that the electroproduction process has a significantly richer phenomenology than the photoproduction process due to the additional longitudinal component of the photon and the $Q^{2}$-dependence of all observables. In our model we will fix the contribution of longitudinal photons to the excitation of the $S_{11}$ resonance by the data of an old Bonn experiment [11], which, of course, has large error bars. The $Q^{2}$-dependence of the resonant part of our electroproduction operator as well as possible longitudinal contributions of other resonances will be treated within the framework of quark models. The non-resonant part of our operator already incorporates the longitudinal components of the transition current $\vec{J}$, and the $Q^{2}$-behaviour is generated by standard dipole form factors.

Fig. E presents the longitudinal cross section $\sigma_{L}$ for kinematics 1 in Table $\mathrm{V}$ in different models, which are more or less consistent with the existing data point. In our standard calculations we will use the non-relativistic constituent quark model with the amplitude of the resonant part of the $L_{0+}$ multipole normalized to the data point from [11]. The reason for the large experimental error bar is due to the fact that the longitudinal excitation of the $S_{11}(1535)$ is weaker than the transverse one. This can be clearly seen in Fig. E. The transverse/longitudinal separation of 
the inclusive cross section, $\sigma_{\text {tot }}=\sigma_{T}+\varepsilon_{L} \sigma_{L}$, leads to a longitudinal cross section $\varepsilon_{L} \sigma_{L}$, which is smaller than the transverse $\sigma_{T}$ by more than an order of magnitude. The data point for the transverse cross section, however, is in good agreement with our model prediction. In this article we will only discuss angular distributions of cross sections that do not require any electron or recoil polarization measurement. The complete formula for the differential cross section describing this scenario is given by (12). For kinematics 1 and 2 in Figs. E, E and E we present the results for the cross sections

$$
\begin{aligned}
& \frac{d \sigma_{T}^{00}}{d \Omega_{\eta}}=\rho R_{T}^{00}, \frac{d \sigma_{L}^{00}}{d \Omega_{\eta}}=\rho R_{L}^{00}, \\
& \frac{d \sigma_{T L}^{00}}{d \Omega_{\eta}}=\rho^{c} R_{T L}^{00}, \frac{d \sigma_{T T}^{00}}{d \Omega_{\eta}}=\rho^{c} R_{T T}^{00}, \\
& \frac{d \sigma_{T T}^{0 x}}{d \Omega_{\eta}}=\rho^{s} R_{T T}^{0 x}, \frac{d \sigma_{T T}^{0 y}}{d \Omega_{\eta}}=\rho^{c} R_{T T}^{0 y},
\end{aligned}
$$

where $\rho=|\vec{k}| / k_{\gamma}^{c m}$.

The resulting transverse cross section $d \sigma_{T}^{00} / d \Omega$ has a rather flat angular distribution as in the photoproduction case. The longitudinal cross section $d \sigma_{L}^{00} / d \Omega$ is smaller than the transverse by about one order of magnitude and has a maximum around $100^{\circ}$ due to the $D_{13}(1520)$ contribution. Of course, the magnitude of this cross section depends strongly on the prediction of the different quark models for $C_{1 / 2}^{p}$. The cross sections $d \sigma_{T T}^{00} / d \Omega$ and $d \sigma_{T L}^{00} / d \Omega$ are very sensitive to the presence of the resonance $D_{13}(1520)$. The transverse-transverse interference cross section almost vanishes without this resonance, and the shape of the transverse-longitudinal interference cross section changes completely when the resonance is decoupled. Finally we will have a look at the cross sections belonging to the response functions ${ }^{c} R_{T T}^{0 y}$ and ${ }^{s} R_{T T}^{0 x}$, which correspond to the photoproduction observables $-P$ and $H$. In electroproduction they could be determined in an experiment with a polarized target or a recoil polarimeter. From photoproduction we already know the sensitivity of these observables to background and Roper resonance contributions. At larger momentum transfer, $Q^{2}=0.120 \mathrm{GeV}^{2}$, we observe different signs for the calculations with and without Roper resonance. An experiment at this kinematics would be invaluable in order to detect a signature of the Roper resonance in eta production. Unfortunately, there are large discrepancies between the different quark models concerning the electromagnetic couplings $A_{1 / 2}^{p}$ and $C_{1 / 2}^{p}$ of the Roper resonance. In addition to this, the Roper resonance value for $A_{1 / 2}^{p}$ in [16] is not fixed very precisely by experiment as well.

TABLE V. Kinematics investigated in electroproduction.

\begin{tabular}{lll}
\hline \hline & Kinematics 1 & Kinematics 2 \\
\hline$\varepsilon_{i}[\mathrm{MeV}]$ & 1337.5 & 989.4 \\
$\varepsilon_{f}[\mathrm{MeV}]$ & 344.5 & 142.2 \\
$\left.\Theta_{e}{ }^{\circ}\right]$ & 55.0 & 55.0 \\
$W[\mathrm{MeV}]$ & 1533.0 & 1533.0 \\
$Q^{2}\left[\mathrm{GeV}^{2}\right]$ & 0.393 & 0.120 \\
$\varepsilon$ & 0.345 & 0.209 \\
\hline \hline
\end{tabular}




\section{CONCLUSION}

Photo- and electroproduction of eta mesons are fastly developing fields in intermediate energy physics. Due to the isospin singlet nature, the eta meson can supply valuable information on the structure of the nucleon complementary to that obtained from pion production. Such experiments are under investigation at various new facilities. The TAPS collaboration has presented results on inclusive and differential photoproduction cross sections off the proton, which were obtained at the MAMI facility, and at the ELSA facility the electroproduction and photoproduction experiments at higher energies are being analysed. Further experiments at these sites are under way with the aim to measure cross sections off the deuteron as well as polarization observables off the proton and the deuteron. In the future, CEBAF will join this experimental field with $\eta$ and $\eta^{\prime}$ electroproduction, investigating various resonance contributions to the process. Last but not the least, the laser-backscattering facility GRAAL is an excellent tool for the investigation of polarization degrees of freedom in eta photoproduction.

In this article we have studied the complete set of response functions for photo- and electroproduction of the eta meson including beam, target and recoil polarization degrees of freedom. Since future experiments will have to be analysed in terms of multipoles, we have also presented the multipole decomposition of the response functions. Near threshold eta production is dominated by the decay of the nucleon resonance $S_{11}(1535)$, which contributes to the $E_{0+}$ and $L_{0+}$ multipoles. We derived the $E_{0+}$ multipole from the latest Mainz [9] and Bonn [12 data for the total photoproduction cross section. The $L_{0+}$ multipole was reconstructed from an old Bonn experiment 11], the $Q^{2}$-dependence of this multipole was investigated within the framework of different quark models [35, 37 39 . The transition current contained the non-resonant background from the model presented in [19] as well as phenomenological resonance contributions. Having fixed the dominating $S$-wave multipoles, we were able to investigate additional resonance contributions of the $P_{11}(1440)$ and $D_{13}(1520)$ resonances. These resonances generate only weak contributions in the unpolarized cross sections. However, they produce tremendous effects in some of the polarization observables by interference with the dominant $S_{11}(1535)$ multipole. We estimated the strength of the Roper and $D_{13}$-resonance multipoles by means of a standard Breit-Wigner approach, having determined the electromagnetic resonance couplings by the helicity elements $A_{1 / 2}^{N}, A_{3 / 2}^{N}$ and $C_{1 / 2}^{N}$, which can be calculated within the framework of quark models. The resonance couplings to the eta meson are determined by the branching fraction $\Gamma_{\eta}(W) / \Gamma(W)$ in our phenomenological model. Our estimate of these additional resonance contributions is compatible with the angular distributions of differential cross sections for photoproduction off the proton measured by the TAPS collaboration. However, it turns out that the measurement of polarization observables can give more reliable information on the resonance contributions. In photoproduction the photon asymmetry should yield precise constraints on the coupling of the $D_{13}(1520)$ to the eta meson. A possible Roper contribution significantly changes the shape of the photoproduction observables $P$ and $H$. These observables are also sensitive to the non-resonant background and provide an additional test of the $\eta N N-$ coupling constant [19]. With regard to photoproduction off the neutron, it would be interesting to see whether the saddle structure in the differential cross section leads to any effects possibly visible in an experiment on the deuteron.

Concerning the electroproduction of eta mesons, our calculations suffer from the large error bars of the existing longitudinal cross sections off the proton used as input. Nevertheless, eta electroproduction is a promising field, because the determination of the $L_{0+}$ multipole from a transverse/longitudinal separation of the total cross section provides a good test of the quark models describing the coupling of a longitudinal photon to the $S_{11}(1535)$ resonance. Furthermore, the separation of the differential cross section $d \sigma_{v} / d \Omega_{\eta}$ into transverse, longitudinal, transverse-transverse and transverse-longitudinal parts can provide additional interesting information on the mechanisms present in eta production. The transverse-transverse interference cross section $d \sigma_{T T}^{00} / d \Omega_{\eta}$ is related to the observable $\Sigma$ in photoproduction. For this reason it also has a characteristic dependence on the $D_{13}$-contribution. The presence of a $D_{13}$-contribution also changes the shape of $d \sigma_{T L}^{00} / d \Omega_{\eta}$ drastically. The Roper and background contributions can be seen best in the response functions ${ }^{s} R_{T T}^{0 x}$ and ${ }^{c} R_{T T}^{0 y}$, whose determination, however, requires a target (or recoil) polarization experiment.

In conclusion eta production exposes a rich phenomenology, which can significantly enlarge our knowledge on the structure of the nucleon. We hope the material we have presented will be useful for the experimental projects under way or even stimulate new experiments to determine some of the polarization observables discussed in this contribution.

\section{APPENDIX A: IDENTICAL RESPONSE FUNCTIONS}

A response function denoted with a star in Table [is identical to another response function via one of the following equations: 


$$
\begin{aligned}
& R_{T}^{00}=-{ }^{c} R_{T T}^{y^{\prime} y}, R_{T}^{0 y}=-{ }^{c} R_{T T}^{y^{\prime} 0}, R_{T}^{y^{\prime} 0}=-{ }^{c} R_{T T}^{0 y}, \\
& R_{T}^{x^{\prime} x}=-{ }^{c} R_{T T}^{z^{\prime} z}, R_{T}^{x^{\prime} z}={ }^{c} R_{T T}^{z^{\prime} x}, R_{T}^{z^{\prime} x}={ }^{c} R_{T T}^{x^{\prime} z}, \\
& R_{T}^{z^{\prime} z}=-{ }^{c} R_{T T}^{x^{\prime} x}, R_{L}^{00}=-R_{L}^{y^{\prime} y}, R_{L}^{0 y}=-R_{L}^{y^{\prime} 0}, \\
& R_{L}^{x^{\prime} x}=-R_{L}^{z^{\prime} z}, R_{L}^{x^{\prime} z}=R_{L}^{z^{\prime} x},{ }^{c} R_{T L}^{00}=-{ }^{c} R_{T L}^{y^{\prime} y}, \\
& { }^{c} R_{T L}^{0 y}=-{ }^{c} R_{T L}^{y^{\prime} 0},{ }^{c} R_{T L}^{x^{\prime} x}=-{ }^{c} R_{T L}^{z^{\prime} z},{ }^{c} R_{T L}^{z^{\prime} x}={ }^{c} R_{T L}^{x^{\prime} z}, \\
& { }^{s} R_{T L}^{0 x}=-{ }^{c} R_{T}^{y^{\prime} z},{ }^{s} R_{T L}^{00}=-{ }^{c} R_{T L^{\prime}}^{y^{\prime} x},{ }^{s} R_{T L}^{x^{\prime} 0}=-{ }^{c} R_{T}^{z^{\prime} y}, \\
& { }^{s} R_{T L}^{z^{\prime} 0}={ }^{c} R_{T L^{\prime}}^{x^{\prime} y},{ }^{c} R_{T T}^{00}=-R_{T}^{y^{\prime} y},{ }^{s} R_{T T}^{0 x}=R_{T T^{\prime}}^{y^{\prime} z}, \\
& { }^{s} R_{T T}^{z^{\prime} 0}=R_{T T^{\prime}}^{x^{\prime} y},{ }^{s} R_{T T}^{0 z}=-R_{T T^{\prime}}^{y^{\prime} x},{ }^{s} R_{T T}^{x^{\prime} 0}=-R_{T T^{\prime}}^{z^{\prime} y}, \\
& { }^{c} R_{T L^{\prime}}^{0 x}=-{ }^{s} R_{T L}^{y^{\prime} z},{ }^{c} R_{T L^{\prime}}^{0 z}={ }^{s} R_{T L}^{y^{\prime} x},{ }^{c} R_{T L^{\prime}}^{x^{\prime} 0}={ }^{s} R_{T L}^{z^{\prime} y}, \\
& { }^{c} R_{T L^{\prime}}^{z^{\prime} 0}=-{ }^{s} R_{T L}^{x^{\prime} y},{ }^{s} R_{T L^{\prime}}^{00}=-{ }^{s} R_{T L^{\prime}}^{y^{\prime} y},{ }^{s} R_{T L^{\prime}}^{0 y}=-{ }^{s} R_{T L^{\prime}}^{y^{\prime} 0}, \\
& { }^{s} R_{T L^{\prime}}^{x^{\prime} x}=-{ }^{s} R_{T}^{z^{\prime} z},{ }^{s} R_{T L^{\prime}}^{z^{\prime} x}={ }^{s} R_{T L^{\prime}}^{x^{\prime} z}, R_{T T^{\prime}}^{0 x}=-{ }^{s} R_{T T}^{y^{\prime} z}, \\
& R_{T T^{\prime}}^{00}={ }^{s} R_{T T}^{y^{\prime} x}, R_{T T^{\prime}}^{x^{\prime} 0}={ }^{s} R_{T T}^{z^{\prime} y}, R_{T T^{\prime}}^{z^{\prime} 0}=-{ }^{s} R_{T T}^{x^{\prime} y} .
\end{aligned}
$$

\section{APPENDIX B: POLARIZATION OBSERVABLES AND RESPONSE FUNCTIONS}

The polarization observables in photoproduction can be expressed in terms of response functions via the following relations:

$$
\begin{aligned}
& d \sigma / d \Omega=\rho R_{T}^{00}, \Sigma=-{ }^{c} R_{T T}^{00} / R_{T}^{00}, \\
& T=R_{T}^{0 y} / R_{T}^{00}, P=R_{T}^{y^{\prime} 0} / R_{T}^{00}, \\
& E=-R_{T T^{\prime}}^{0 z} / R_{T}^{00}, F=R_{T T^{\prime}}^{0 x} / R_{T}^{00}, \\
& G=-{ }^{s} R_{T T}^{0 z} / R_{T}^{00}, H={ }^{s} R_{T T}^{0 x} / R_{T}^{00}, \\
& O_{x^{\prime}}={ }^{s} R_{T T}^{x^{\prime} 0} / R_{T}^{00}, O_{z^{\prime}}={ }^{s} R_{T T}^{z^{\prime} 0} / R_{T}^{00}, \\
& C_{x^{\prime}}=-R_{T T^{\prime}}^{x^{\prime}} / R_{T}^{00}, C_{z^{\prime}}=-R_{T T^{\prime}}^{z^{\prime}} / R_{T}^{00}, \\
& T_{x^{\prime}}=R_{T}^{x^{\prime} x} / R_{T}^{00}, T_{z^{\prime}}=R_{T}^{z^{\prime} x} / R_{T}^{00}, \\
& L_{x^{\prime}}=-R_{T}^{x^{\prime} z} / R_{T}^{00}, L_{z^{\prime}}=R_{T}^{z^{\prime} z} / R_{T}^{00} .
\end{aligned}
$$

\section{APPENDIX C: RESPONSE FUNCTIONS AND CGLN AMPLITUDES}

$$
\begin{aligned}
R_{T}^{00}= & \left|F_{1}\right|^{2}+\left|F_{2}\right|^{2}+\frac{\sin ^{2} \Theta}{2}\left(\left|F_{3}\right|^{2}+\left|F_{4}\right|^{2}\right) \\
& +\operatorname{Re}\left\{\sin ^{2} \Theta\left(F_{2}^{*} F_{3}+F_{1}^{*} F_{4}+\cos \Theta F_{3}^{*} F_{4}\right)\right. \\
& \left.-2 \cos \Theta F_{1}^{*} F_{2}\right\}, \\
R_{T}^{0 y}= & \operatorname{Im}\left\{\operatorname { s i n } \Theta \left(F_{1}^{*} F_{3}-F_{2}^{*} F_{4}+\cos \Theta\left(F_{1}^{*} F_{4}-F_{2}^{*} F_{3}\right)\right.\right. \\
& \left.\left.-\sin ^{2} \Theta F_{3}^{*} F_{4}\right)\right\}, \\
R_{T}^{y^{\prime} 0}= & \operatorname{Im}\left\{\operatorname { s i n } \Theta \left(-2 F_{1}^{*} F_{2}-F_{1}^{*} F_{3}+F_{2}^{*} F_{4}\right.\right. \\
& \left.\left.+\cos \Theta\left(F_{2}^{*} F_{3}-F_{1}^{*} F_{4}\right)+\sin ^{2} \Theta F_{3}^{*} F_{4}\right)\right\}, \\
R_{T}^{x^{\prime} x}= & \operatorname{Re}\left\{\operatorname { s i n } ^ { 2 } \Theta \left(-F_{1}^{*} F_{3}-F_{2}^{*} F_{4}-F_{3}^{*} F_{4}\right.\right. \\
& \left.\left.-\frac{1}{2} \cos \Theta\left(\left|F_{3}\right|^{2}+\left|F_{4}\right|^{2}\right)\right)\right\}, \\
R_{T}^{x^{\prime} z}= & \left\{\operatorname { s i n } \Theta \left(\left|F_{1}\right|^{2}-\left|F_{2}\right|^{2}+\frac{1}{2} \sin ^{2} \Theta\left(\left|F_{4}\right|^{2}-\left|F_{3}\right|^{2}\right)\right.\right.
\end{aligned}
$$




$$
\begin{aligned}
& \left.\left.-F_{2}^{*} F_{3}+F_{1}^{*} F_{4}+\cos \Theta\left(F_{1}^{*} F_{3}-F_{2}^{*} F_{4}\right)\right)\right\}, \\
& R_{T}^{z^{\prime} x}=\operatorname{Re}\left\{\operatorname { s i n } \Theta \left(-F_{2}^{*} F_{3}+F_{1}^{*} F_{4}+\cos \Theta\left(F_{1}^{*} F_{3}-F_{2}^{*} F_{4}\right)\right.\right. \\
& \left.\left.+\frac{1}{2} \sin ^{2} \Theta\left(\left|F_{4}\right|^{2}-\left|F_{3}\right|^{2}\right)\right)\right\}, \\
& R_{T}^{z^{\prime} z}=\operatorname{Re}\left\{2 F_{1}^{*} F_{2}-\cos \Theta\left(\left|F_{1}\right|^{2}+\left|F_{2}\right|^{2}\right)\right. \\
& +\sin ^{2} \Theta\left(F_{1}^{*} F_{3}+F_{2}^{*} F_{4}+F_{3}^{*} F_{4}\right) \\
& \left.+\frac{1}{2} \cos \Theta \sin ^{2} \Theta\left(\left|F_{3}\right|^{2}+\left|F_{4}\right|^{2}\right)\right\}, \\
& R_{L}^{00}=\operatorname{Re}\left\{\left|F_{5}\right|^{2}+\left|F_{6}\right|^{2}+2 \cos \Theta F_{5}^{*} F_{6}\right\}, \\
& R_{L}^{0 y}=-2 \sin \Theta \operatorname{Im}\left\{F_{5}^{*} F_{6}\right\} \text {, } \\
& R_{L}^{x^{\prime} x}=\operatorname{Re}\left\{-2 F_{5}^{*} F_{6}-\cos \Theta\left(\left|F_{5}\right|^{2}+\left|F_{6}\right|^{2}\right)\right\}, \\
& R_{L}^{z^{\prime} x}=\sin \Theta\left(\left|F_{6}\right|^{2}-\left|F_{5}\right|^{2}\right) \text {, } \\
& { }^{c} R_{T L}^{00}=\sin \Theta \operatorname{Re}\left\{-F_{2}^{*} F_{5}-F_{3}^{*} F_{5}-F_{1}^{*} F_{6}-F_{4}^{*} F_{6}\right. \\
& \left.-\cos \Theta\left(F_{4}^{*} F_{5}+F_{3}^{*} F_{6}\right)\right\} \text {, } \\
& { }^{s} R_{T L}^{0 x}=\operatorname{Im}\left\{-F_{1}^{*} F_{5}+F_{2}^{*} F_{6}+\cos \Theta\left(F_{2}^{*} F_{5}-F_{1}^{*} F_{6}\right)\right\}, \\
& { }^{c} R_{T L}^{0 y}=\operatorname{Im}\left\{-F_{1}^{*} F_{5}+F_{2}^{*} F_{6}+\cos \Theta\left(F_{2}^{*} F_{5}-F_{1}^{*} F_{6}\right)\right. \\
& \left.+\sin ^{2} \Theta\left(F_{3}^{*} F_{6}-F_{4}^{*} F_{5}\right)\right\} \text {, } \\
& { }^{s} R_{T L}^{0 z}=\sin \Theta \operatorname{Im}\left\{F_{2}^{*} F_{5}+F_{1}^{*} F_{6}\right\}, \\
& { }^{s} R_{T L}^{x^{\prime} 0}=\operatorname{Im}\left\{-F_{2}^{*} F_{5}+F_{1}^{*} F_{6}+\cos \Theta\left(F_{1}^{*} F_{5}-F_{2}^{*} F_{6}\right)\right\}, \\
& { }^{s} R_{T L}^{z^{\prime} 0}=\sin \Theta \operatorname{Im}\left\{F_{1}^{*} F_{5}+F_{2}^{*} F_{6}\right\}, \\
& { }^{c} R_{T L}^{x^{\prime} x}=\sin \Theta \operatorname{Re}\left\{F_{1}^{*} F_{5}+F_{4}^{*} F_{5}+F_{2}^{*} F_{6}+F_{3}^{*} F_{6}\right. \\
& \left.+\cos \Theta\left(F_{3}^{*} F_{5}+F_{4}^{*} F_{6}\right)\right\} \text {, } \\
& { }^{c} R_{T L}^{z^{\prime} x}=\operatorname{Re}\left\{F_{2}^{*} F_{5}-F_{1}^{*} F_{6}+\cos \Theta\left(F_{2}^{*} F_{6}-F_{1}^{*} F_{5}\right)\right. \\
& \left.+\sin ^{2} \Theta\left(F_{3}^{*} F_{5}-F_{4}^{*} F_{6}\right)\right\} \text {, } \\
& { }^{c} R_{T T}^{00}=\frac{1}{2} \sin ^{2} \Theta\left\{\left|F_{3}\right|^{2}+\left|F_{4}\right|^{2}\right\} \\
& +\sin ^{2} \Theta \operatorname{Re}\left\{F_{2}^{*} F_{3}+F_{1}^{*} F_{4}+\cos \Theta F_{3}^{*} F_{4}\right\}, \\
& { }^{s} R_{T T}^{0 x}=\sin \Theta \operatorname{Im}\left\{2 F_{1}^{*} F_{2}+F_{1}^{*} F_{3}-F_{2}^{*} F_{4}\right. \\
& \left.+\cos \Theta\left(F_{1}^{*} F_{4}-F_{2}^{*} F_{3}\right)\right\} \text {, } \\
& { }^{s} R_{T T}^{0 z}=-\sin ^{2} \Theta \operatorname{Im}\left\{F_{2}^{*} F_{3}+F_{1}^{*} F_{4}\right\}, \\
& { }^{s} R_{T T}^{x^{\prime} 0}=\sin \Theta \operatorname{Im}\left\{F_{2}^{*} F_{3}-F_{1}^{*} F_{4}+\cos \Theta\left(F_{2}^{*} F_{4}-F_{1}^{*} F_{3}\right)\right\}, \\
& { }^{s} R_{T T}^{z^{\prime} 0}=-\sin ^{2} \Theta \operatorname{Im}\left\{F_{1}^{*} F_{3}+F_{2}^{*} F_{4}\right\}, \\
& { }^{s} R_{T L^{\prime}}^{00}=-\sin \Theta \operatorname{Im}\left\{F_{2}^{*} F_{5}+F_{3}^{*} F_{5}+F_{1}^{*} F_{6}+F_{4}^{*} F_{6}\right. \\
& \left.+\cos \Theta\left(F_{4}^{*} F_{5}+F_{3}^{*} F_{6}\right)\right\} \text {, } \\
& { }^{c} R_{T L^{\prime}}^{0 x}=\operatorname{Re}\left\{-F_{1}^{*} F_{5}+F_{2}^{*} F_{6}+\cos \Theta\left(F_{2}^{*} F_{5}-F_{1}^{*} F_{6}\right)\right\}, \\
& { }^{s} R_{T L^{\prime}}^{0 y}=\operatorname{Re}\left\{F_{1}^{*} F_{5}-F_{2}^{*} F_{6}+\cos \Theta\left(F_{1}^{*} F_{6}-F_{2}^{*} F_{5}\right)\right. \\
& \left.+\sin ^{2} \Theta\left(F_{4}^{*} F_{5}-F_{3}^{*} F_{6}\right)\right\} \text {, } \\
& { }^{c} R_{T L^{\prime}}^{0 z}=\sin \Theta \operatorname{Re}\left\{F_{2}^{*} F_{5}+F_{1}^{*} F_{6}\right\}, \\
& { }^{c} R_{T L^{\prime}}^{x^{\prime}}=\operatorname{Re}\left\{-F_{2}^{*} F_{5}+F_{1}^{*} F_{6}+\cos \Theta\left(F_{1}^{*} F_{5}-F_{2}^{*} F_{6}\right)\right\}, \\
& { }^{c} R_{T L^{\prime}}^{z^{\prime} 0}=\sin \Theta \operatorname{Re}\left\{F_{1}^{*} F_{5}+F_{2}^{*} F_{6}\right\}, \\
& { }^{s} R_{T L^{\prime}}^{x^{\prime} x}=\sin \Theta \operatorname{Im}\left\{F_{1}^{*} F_{5}+F_{4}^{*} F_{5}+F_{2}^{*} F_{6}+F_{3}^{*} F_{6}\right. \\
& \left.+\cos \Theta\left(F_{3}^{*} F_{5}+F_{4}^{*} F_{6}\right)\right\} \text {, } \\
& { }^{s} R_{T L^{\prime}}^{z^{\prime} x}=\operatorname{Im}\left\{F_{2}^{*} F_{5}-F_{1}^{*} F_{6}+\cos \Theta\left(-F_{1}^{*} F_{5}+F_{2}^{*} F_{6}\right)\right. \\
& \left.+\sin ^{2} \Theta\left(F_{3}^{*} F_{5}-F_{4}^{*} F_{6}\right)\right\} \text {, }
\end{aligned}
$$




$$
\begin{aligned}
R_{T T^{\prime}}^{0 x}= & \sin \Theta \operatorname{Re}\left\{F_{1}^{*} F_{3}-F_{2}^{*} F_{4}+\cos \Theta\left(-F_{2}^{*} F_{3}+F_{1}^{*} F_{4}\right)\right\}, \\
R_{T T^{\prime}}^{0 z}= & -\left|F_{1}\right|^{2}-\left|F_{2}\right|^{2} \\
& +\operatorname{Re}\left\{2 \cos \Theta F_{1}^{*} F_{2}-\sin ^{2} \Theta\left(F_{2}^{*} F_{3}+F_{1}^{*} F_{4}\right)\right\}, \\
R_{T T^{\prime}}^{x^{\prime} 0}= & \sin \Theta \operatorname{Re}\left\{-\left|F_{1}\right|^{2}+\left|F_{2}\right|^{2}+F_{2}^{*} F_{3}\right. \\
& \left.-F_{1}^{*} F_{4}+\cos \Theta\left(F_{2}^{*} F_{4}-F_{1}^{*} F_{3}\right)\right\}, \\
R_{T T^{\prime}}^{z^{\prime} 0}= & \operatorname{Re}\left\{-2 F_{1}^{*} F_{2}+\cos \Theta\left(\left|F_{1}\right|^{2}+\left|F_{2}\right|^{2}\right)\right. \\
& \left.-\sin ^{2} \Theta\left(F_{1}^{*} F_{3}+F_{2}^{*} F_{4}\right)\right\} .
\end{aligned}
$$

\section{APPENDIX D: RESPONSE FUNCTIONS AND HELICITY AMPLITUDES}

$$
\begin{aligned}
& R_{T}^{00}=\frac{1}{2}\left(\left|H_{1}\right|^{2}+\left|H_{2}\right|^{2}+\left|H_{3}\right|^{2}+\left|H_{4}\right|^{2}\right), \\
& R_{T}^{0 y}=-\operatorname{Im}\left\{H_{1}^{*} H_{2}+H_{3}^{*} H_{4}\right\}, \\
& R_{T}^{y^{\prime} 0}=\operatorname{Im}\left\{H_{1}^{*} H_{3}+H_{2}^{*} H_{4}\right\}, \\
& R_{T}^{x^{\prime} x}=\operatorname{Re}\left\{H_{1}^{*} H_{4}+H_{2}^{*} H_{3}\right\}, \\
& R_{T}^{x^{\prime} z}=\operatorname{Re}\left\{H_{1}^{*} H_{3}-H_{2}^{*} H_{4}\right\}, \\
& R_{T}^{z^{\prime} x}=\operatorname{Re}\left\{H_{1}^{*} H_{2}-H_{3}^{*} H_{4}\right\}, \\
& R_{T}^{z^{\prime} z}=\frac{1}{2}\left(\left|H_{1}\right|^{2}-\left|H_{2}\right|^{2}-\left|H_{3}\right|^{2}+\left|H_{4}\right|^{2}\right), \\
& R_{L}^{00}=\left|H_{5}\right|^{2}+\left|H_{6}\right|^{2}, \\
& R_{L}^{0 y}=-2 \operatorname{Im}\left\{H_{5}^{*} H_{6}\right\}, \\
& R_{L}^{x^{\prime} x}=-\left|H_{5}\right|^{2}+\left|H_{6}\right|^{2}, \\
& R_{L}^{z^{\prime} x}=2 \operatorname{Re}\left\{H_{5}^{*} H_{6}\right\} \text {, } \\
& { }^{c} R_{T L}^{00}=\frac{1}{\sqrt{2}} \operatorname{Re}\left\{H_{5}^{*} H_{1}-H_{5}^{*} H_{4}+H_{6}^{*} H_{2}+H_{6}^{*} H_{3}\right\}, \\
& { }^{s} R_{T L}^{0 x}=\frac{1}{\sqrt{2}} \operatorname{Im}\left\{-H_{5}^{*} H_{2}+H_{5}^{*} H_{3}-H_{6}^{*} H_{1}-H_{6}^{*} H_{4}\right\}, \\
& { }^{c} R_{T L}^{0 y}=\frac{1}{\sqrt{2}} \operatorname{Im}\left\{-H_{5}^{*} H_{2}-H_{5}^{*} H_{3}+H_{6}^{*} H_{1}-H_{6}^{*} H_{4}\right\}, \\
& { }^{s} R_{T L}^{0 z}=\frac{1}{\sqrt{2}} \operatorname{Im}\left\{-H_{5}^{*} H_{1}-H_{5}^{*} H_{4}+H_{6}^{*} H_{2}-H_{6}^{*} H_{3}\right\}, \\
& { }^{s} R_{T L}^{x^{\prime} 0}=\frac{1}{\sqrt{2}} \operatorname{Im}\left\{H_{5}^{*} H_{2}-H_{5}^{*} H_{3}-H_{6}^{*} H_{1}-H_{6}^{*} H_{4}\right\}, \\
& { }^{s} R_{T L}^{z^{\prime} 0}=\frac{1}{\sqrt{2}} \operatorname{Im}\left\{-H_{5}^{*} H_{1}-H_{5}^{*} H_{4}-H_{6}^{*} H_{2}+H_{6}^{*} H_{3}\right\}, \\
& { }^{c} R_{T L}^{x^{\prime} x}=\frac{1}{\sqrt{2}} \operatorname{Re}\left\{-H_{5}^{*} H_{1}+H_{5}^{*} H_{4}+H_{6}^{*} H_{2}+H_{6}^{*} H_{3}\right\}, \\
& { }^{c} R_{T L}^{z^{\prime} x}=\frac{1}{\sqrt{2}} \operatorname{Re}\left\{H_{5}^{*} H_{2}+H_{5}^{*} H_{3}+H_{6}^{*} H_{1}-H_{6}^{*} H_{4}\right\}, \\
& { }^{c} R_{T T}^{00}=\operatorname{Re}\left\{-H_{1}^{*} H_{4}+H_{2}^{*} H_{3}\right\}, \\
& { }^{s} R_{T T}^{0 x}=\operatorname{Im}\left\{H_{1}^{*} H_{3}-H_{2}^{*} H_{4}\right\}, \\
& { }^{s} R_{T T}^{0 z}=-\operatorname{Im}\left\{H_{1}^{*} H_{4}+H_{2}^{*} H_{3}\right\} \text {, } \\
& { }^{s} R_{T T}^{x^{\prime} 0}=\operatorname{Im}\left\{H_{1}^{*} H_{2}-H_{3}^{*} H_{4}\right\}, \\
& { }^{s} R_{T T}^{z^{\prime} 0}=\operatorname{Im}\left\{-H_{1}^{*} H_{4}+H_{2}^{*} H_{3}\right\}, \\
& { }^{s} R_{T L^{\prime}}^{00}=\frac{1}{\sqrt{2}} \operatorname{Im}\left\{-H_{5}^{*} H_{1}+H_{5}^{*} H_{4}-H_{6}^{*} H_{2}-H_{6}^{*} H_{3}\right\},
\end{aligned}
$$




$$
\begin{aligned}
{ }^{c} R_{T L^{\prime}}^{0 x} & =\frac{1}{\sqrt{2}} \operatorname{Re}\left\{H_{5}^{*} H_{2}-H_{5}^{*} H_{3}+H_{6}^{*} H_{1}+H_{6}^{*} H_{4}\right\}, \\
{ }^{s} R_{T L^{\prime}}^{0 y} & =\frac{1}{\sqrt{2}} \operatorname{Re}\left\{-H_{5}^{*} H_{2}-H_{5}^{*} H_{3}+H_{6}^{*} H_{1}-H_{6}^{*} H_{4}\right\}, \\
{ }^{c} R_{T L^{\prime}}^{0 z} & =\frac{1}{\sqrt{2}} \operatorname{Re}\left\{H_{5}^{*} H_{1}+H_{5}^{*} H_{4}-H_{6}^{*} H_{2}+H_{6}^{*} H_{3}\right\}, \\
{ }^{c} R_{T L^{\prime}}^{x^{\prime} 0} & =\frac{1}{\sqrt{2}} \operatorname{Re}\left\{-H_{5}^{*} H_{2}+H_{5}^{*} H_{3}+H_{6}^{*} H_{1}+H_{6}^{*} H_{4}\right\}, \\
{ }^{c} R_{T L^{\prime}}^{z^{\prime} 0} & =\frac{1}{\sqrt{2}} \operatorname{Re}\left\{H_{5}^{*} H_{1}+H_{5}^{*} H_{4}+H_{6}^{*} H_{2}-H_{6}^{*} H_{3}\right\}, \\
{ }^{s} R_{T L^{\prime}}^{x^{\prime} x} & =\frac{1}{\sqrt{2}} \operatorname{Im}\left\{H_{5}^{*} H_{1}-H_{5}^{*} H_{4}-H_{6}^{*} H_{2}-H_{6}^{*} H_{3}\right\}, \\
{ }^{s} R_{T L^{\prime}}^{z^{\prime} x} & =\frac{1}{\sqrt{2}} \operatorname{Im}\left\{-H_{5}^{*} H_{2}-H_{5}^{*} H_{3}-H_{6}^{*} H_{1}+H_{6}^{*} H_{4}\right\}, \\
R_{T T^{\prime}}^{0 x} & =\operatorname{Re}\left\{H_{1}^{*} H_{2}+H_{3}^{*} H_{4}\right\}, \\
R_{T T^{\prime}}^{0 z} & =\frac{1}{2}\left(\left|H_{1}\right|^{2}-\left|H_{2}\right|^{2}+\left|H_{3}\right|^{2}-\left|H_{4}\right|^{2}\right), \\
R_{T T^{\prime}}^{x^{\prime} 0} & =\operatorname{Re}\left\{H_{1}^{*} H_{3}+H_{2}^{*} H_{4}\right\}, \\
R_{T T^{\prime}}^{z^{\prime} 0} & =\frac{1}{2}\left(\left|H_{1}\right|^{2}+\left|H_{2}\right|^{2}-\left|H_{3}\right|^{2}-\left|H_{4}\right|^{2}\right) .
\end{aligned}
$$

\section{APPENDIX E: MULTIPOLE EXPANSION OF RESPONSE FUNCTIONS FOR ETA ELECTROPRODUCTION}

In the following we have kept all terms proportional to the dominant multipoles $E_{0+}$ and $L_{0+}$ and interference terms with the smaller multipoles considered in the text, $M_{1-}, L_{1-}, E_{2-}, M_{2-}$ and $L_{2-}$.

$$
\begin{aligned}
R_{T}^{00}=\left|E_{0+}\right|^{2}-\operatorname{Re}\left\{E _ { 0 + } ^ { * } \left[2 \cos \Theta M_{1-}\right.\right. & \left.\left.-\left(3 \cos ^{2} \Theta-1\right)\left(E_{2-}-3 M_{2-}\right)\right]\right\}, \\
R_{T}^{0 y}= & -3 \sin \Theta \cos \Theta \operatorname{Im}\left\{E_{0+}^{*}\left(E_{2-}+M_{2-}\right)\right\}, \\
R_{T}^{y^{\prime} 0}= & -\sin \Theta \operatorname{Im}\left\{E_{0+}^{*}\left[2 M_{1-}-3 \cos \Theta\left(E_{2-}-3 M_{2-}\right)\right]\right\}, \\
R_{T}^{x^{\prime} x}= & 0, \\
R_{T}^{x^{\prime} z}= & \sin \Theta\left[\left|E_{0+}\right|^{2}-\operatorname{Re}\left\{E_{0+}\left(E_{2-}-3 M_{2-}\right)\right\}\right], \\
R_{T}^{z^{\prime} x}= & -3 \sin \Theta \operatorname{Re}\left\{E_{0+}^{*}\left(E_{2-}+M_{2-}\right)\right\}, \\
R_{T}^{z^{\prime} z}= & -\cos \Theta\left|E_{0+}\right|^{2}+2 \operatorname{Re}\left\{E _ { 0 + } ^ { * } \left[M_{1-}\right.\right. \\
& \left.\left.-\cos \Theta\left(E_{2-}-3 M_{2-}\right)\right]\right\} \\
R_{L}^{00}= & \left.L_{0+}\right|^{2}+2 \operatorname{Re}\left\{L _ { 0 + } ^ { * } \left(\cos \Theta L_{1-}\right.\right. \\
& \left.\left.-2\left(1-3 \cos ^{2} \Theta\right) L_{2-}\right)\right\}, \\
R_{L}^{0 y}= & -2 \sin \Theta \operatorname{Im}\left\{L_{0+}^{*}\left(L_{1-}+6 \cos \Theta L_{2-}\right)\right\}, \\
R_{L}^{x^{\prime} x}= & -\cos \Theta\left|L_{0+}\right|^{2}-2 \operatorname{Re}\left\{L_{0+}^{*}\left(L_{1-}+4 \cos \Theta L_{2-}\right)\right\}, \\
R_{L}^{z^{\prime} x}= & -\sin \Theta\left(\left|L_{0+}\right|^{2}+4 \operatorname{Re}\left\{L_{0+}^{*} L_{2-}\right\}\right), \\
{ }^{c} R_{T L}^{00}= & -\sin \Theta \operatorname{Re}\left\{E_{0+}^{*}\left(L_{1-}+6 \cos \Theta L_{2-}\right)\right. \\
& \left.+L_{0+}^{*}\left(M_{1-}+3 \cos \Theta\left(M_{2-}-E_{2-}\right)\right)\right\}, \\
{ }^{s} R_{T L}^{0 x}= & \operatorname{Im}\left\{L_{0+}^{*} E_{0+}+E_{0+}^{*}\left(-\cos \Theta L_{1-}\right.\right. \\
& \left.+2\left(1-3 \cos ^{2} \Theta L_{2-}\right)\right)+L_{0+}^{*}\left(-\cos \Theta M_{1-}\right. \\
& \left.\left.+E_{2-}+3\left(1-2 \cos { }^{2} \Theta\right) M_{2-}\right)\right\},
\end{aligned}
$$




$$
\begin{aligned}
& { }^{c} R_{T L}^{0 y}=\operatorname{Im}\left\{L_{0+}^{*} E_{0+}+E_{0+}^{*}\left(-\cos \Theta L_{1-}\right.\right. \\
& \left.+2\left(1-3 \cos ^{2} \Theta\right) L_{2-}\right)+L_{0+}^{*}\left(-\cos \Theta M_{1-}\right. \\
& \left.\left.+\left(3 \cos ^{2} \Theta-2\right) E_{2-}-3 \cos ^{2} \Theta M_{2-}\right)\right\}, \\
& { }^{s} R_{T L}^{0 z}=\sin \Theta \operatorname{Im}\left\{E_{0+}^{*}\left(L_{1-}+6 \cos \Theta L_{2-}\right)\right. \\
& \left.-L_{0+}^{*}\left(M_{1-}+6 \cos \Theta M_{2-}\right)\right\} \text {, } \\
& { }^{s} R_{T L}^{x^{\prime} 0}=\operatorname{Im}\left\{-\cos \Theta L_{0+}^{*} E_{0+}+E_{0+}^{*}\left(L_{1-}+4 \cos \Theta L_{2-}\right)\right. \\
& \left.+L_{0+}^{*}\left(M_{1-}+\cos \Theta\left(3 M_{2-}-E_{2-}\right)\right)\right\}, \\
& { }^{s} R_{T L}^{z^{\prime} 0}=-\sin \Theta \operatorname{Im}\left\{L_{0+}^{*} E_{0+}+2 E_{0+}^{*} L_{2-}\right. \\
& \left.+L_{0+}^{*}\left(E_{2-}+3 M_{2-}\right)\right\} \text {, } \\
& { }^{c} R_{T L}^{x^{\prime} x}=\sin \Theta \operatorname{Re}\left\{L_{0+} E_{0+}-2 E_{0+} L_{2-}-2 L_{0+} E_{2-}\right\}, \\
& { }^{c} R_{T L}^{z^{\prime} x}=\operatorname{Re}\left\{-\cos \Theta L_{0+}^{*} E_{0+}-E_{0+}^{*}\left(L_{1-}+4 \cos \Theta L_{2-}\right)\right. \\
& \left.+L_{0+}^{*}\left(M_{1-}-\cos \Theta\left(E_{2-}-3 M_{2-}\right)\right)\right\}, \\
& { }^{c} R_{T T}^{00}=-3 \sin ^{2} \Theta \operatorname{Re}\left\{E_{0+}^{*}\left(E_{2-}+M_{2-}\right)\right\}, \\
& { }^{s} R_{T T}^{0 x}=\sin \Theta \operatorname{Im}\left\{E_{0+}^{*}\left[2 M_{1-}-3 \cos \Theta\left(E_{2-}-3 M_{2-}\right)\right]\right\}, \\
& { }^{s} R_{T T}^{0 z}=3 \sin ^{2} \Theta \operatorname{Im}\left\{E_{0+}^{*}\left(E_{2-}+M_{2-}\right)\right\}, \\
& { }^{s} R_{T T}^{x^{\prime} 0}=3 \sin \Theta \operatorname{Im}\left\{E_{0+}^{*}\left(E_{2-}+M_{2-}\right)\right\}, \\
& { }^{s} R_{T T}^{z^{\prime} 0}=0, \\
& { }^{s} R_{T L^{\prime}}^{00}=\sin \Theta \operatorname{Im}\left\{E_{0+}^{*}\left(-L_{1-}-6 \cos \Theta L_{2-}\right)\right. \\
& \left.+L_{0+}^{*}\left(M_{1-}+3 \cos \Theta\left(M_{2-}-E_{2-}\right)\right)\right\}, \\
& { }^{c} R_{T L^{\prime}}^{0 x}=\operatorname{Re}\left\{-L_{0+}^{*} E_{0+}+E_{0+}^{*}\left(-\cos \Theta L_{1-}\right.\right. \\
& \left.+2\left(1-3 \cos ^{2} \Theta\right) L_{2-}\right) \\
& \left.+L_{0+}^{*}\left(\cos \Theta M_{1-}-3\left(1-2 \cos ^{2} \Theta\right) M_{2-}-E_{2-}\right)\right\}, \\
& { }^{s} R_{T L^{\prime}}^{0 y}=\operatorname{Re}\left\{L_{0+}^{*} E_{0+}+E_{0+}^{*}\left(\cos \Theta L_{1-}\right.\right. \\
& \left.-2\left(1-3 \cos ^{2} \Theta\right) L_{2-}\right) \\
& +L_{0+}^{*}\left(-\cos \Theta M_{1-}+\left(1-3 \sin ^{2} \Theta\right) E_{2-}\right. \\
& \left.\left.-3 \cos ^{2} \Theta M_{2-}\right)\right\} \text {, } \\
& { }^{c} R_{T L^{\prime}}^{0 z}=\sin \Theta \operatorname{Re}\left\{E_{0+}^{*}\left(L_{1-}+6 \cos \Theta L_{2-}\right)\right. \\
& \left.+L_{0+}^{*}\left(M_{1-}+6 \cos \Theta M_{2-}\right)\right\}, \\
& { }^{c} R_{T L^{\prime}}^{x^{\prime} 0}=\operatorname{Re}\left\{\cos \Theta L_{0+}^{*} E_{0+}+E_{0+}^{*}\left(L_{1-}+4 \cos \Theta L_{2-}\right)\right. \\
& \left.+L_{0+}^{*}\left(-M_{1-}+\cos \Theta\left(E_{2-}-3 M_{2-}\right)\right)\right\}, \\
& { }^{c} R_{T L^{\prime}}^{z^{\prime} 0}=\sin \Theta \operatorname{Re}\left\{L_{0+}^{*} E_{0+}-2 E_{0+}^{*} L_{2-}\right. \\
& \left.+L_{0+}\left(E_{2-}+3 M_{2-}\right)\right\} \text {, } \\
& { }^{s} R_{T L^{\prime}}^{x^{\prime} x}=\sin \Theta \operatorname{Im}\left\{-L_{0+}^{*} E_{0+}-2 E_{0+}^{*} L_{2-}+2 L_{0+}^{*} E_{2-}\right\}, \\
& { }^{s} R_{T L^{\prime}}^{z^{\prime} x}=\operatorname{Im}\left\{\cos \Theta L_{0+}^{*} E_{0+}+E_{0+}^{*}\left(-L_{1-}-4 \cos \Theta L_{2-}\right)\right. \\
& \left.+L_{0+}^{*}\left(-M_{1-}+\cos \Theta\left(E_{2-}-3 M_{2-}\right)\right)\right\}, \\
& R_{T T^{\prime}}^{0 x}=-3 \sin \Theta \cos \Theta \operatorname{Re}\left\{E_{0+}^{*}\left(E_{2-}+M_{2-}\right)\right\}, \\
& R_{T T^{\prime}}^{0 z}=-\left|E_{0+}\right|^{2}+\operatorname{Re}\left\{E _ { 0 + } ^ { * } \left[2 \cos \Theta M_{1-}\right.\right. \\
& \left.\left.-\left(3 \cos ^{2} \Theta-1\right)\left(E_{2-}-3 M_{2-}\right)\right]\right\} \text {, } \\
& R_{T T^{\prime}}^{x^{\prime} 0}=-\sin \Theta\left[\left|E_{0+}\right|^{2}-\operatorname{Re}\left\{E_{0+}^{*}\left(E_{2-}-3 M_{2-}\right)\right\}\right], \\
& R_{T T^{\prime}}^{z^{\prime} 0}=\cos \Theta\left|E_{0+}\right|^{2}-2 \operatorname{Re}\left\{E _ { 0 + } ^ { * } \left[M_{1-}\right.\right. \\
& \left.\left.-\cos \Theta\left(E_{2-}-3 M_{2-}\right)\right]\right\} \text {. }
\end{aligned}
$$


[1] B. Delcourt et al.: Phys. Lett. B 29 (1967) 75

[2] C. Bacci et al.: Phys. Rev. Lett. 20 (1968) 571; Nuovo Cimento 45 (1966) 983

[3] Aachen-Berlin-Bonn-Hamburg-München Collaboration: Phys. Rev. 175 (1968) 1669

[4] Cambridge Bubble Chamber Group: Phys. Rev. 169 (1968) 1081

[5] E. D. Bloom et al.: Phys. Rev. Lett. 21 (1968) 1100

[6] C. A. Heusch et al.: Phys. Rev. Lett. 25 (1970) 1381

[7] S. Dytman et al.: Bull. Amer. Phys. Soc. 35 (1990) 1679; Contribution to the Fourth Conf. on the intersection of particle and nuclear physics, Tucson 1991

[8] S. Homma et al.: J. Phys. Soc. Jpn. 57 (1988) 828

[9] B. Krusche et al.: Phys. Rev. Lett., in print; private communication

[10] M. Breuer: Thesis (Bonn University, 1994)

[11] H. Breuker et al.: Phys. Lett. 74 B (1978) 409; C. Nietzel: Thesis (Bonn University, 1978)

[12] M. Wilhelm: Thesis (Bonn University, 1993)

[13] S. Dytman et al.: CEBAF PR 89-039 B.G. Ritchie et al.: CEBAF PR 91-008 M.F. Vineyard et al.: CEBAF PR 93-008 B.G. Ritchie et al.: CEBAF PR 94-008 W. Bertozzi et al.: CEBAF PR 94-119

[14] N. Isgur, G. Karl: Phys. Rev. D 18 (1978) 4187; N. Isgur, G. Karl: Phys. Rev. D 19 (1979) 2653; N. Isgur, G. Karl, R. Koniuk: Phys. Rev. D 25 (1982) 2394

[15] Particle Data Group: Phys. Rev. D 50 I (1994) 1173

[16] Particle Data Group: Phys. Rev. D 45 II (1992) I.1

[17] G. Höhler, A. Schulte: $\pi N$ Newsletter 7 (1992) 94

[18] G. Höhler: $\pi N$ Newsletter 9 (1993) 1

[19] L. Tiator, C. Bennhold, S. S. Kamalov: Nucl. Phys. A 580 (1994) 455

[20] V. Bernard et al.: Phys. Lett. B 268 (1991) 291; V. Bernard, N. Kaiser, U.-G. Meissner: Strasbourg preprint, CRN 94-62

[21] H. R. Hicks et al.: Phys. Rev D 7 (1973) 2614

[22] M. Benmerrouche, N. C. Mukhopadhyay: Phys. Rev. Lett. 67 (1991) 1070

[23] C. Bennhold, H. Tanabe: Nucl. Phys. A 530 (1991) 625

[24] Ch. Sauermann, B. L. Friman, W. Nörenberg: Phys. Lett. B 341 (1995) 261

[25] T. Feuster: Diploma Thesis (Gießen University, 1993)

[26] J. Denschlag: Diploma Thesis, KPH 19/94 (Mainz University, 1994)

[27] J. D. Bjorken, S. D. Drell: Relativistic Quantum Mechanics. New York: McGraw-Hill 1964

[28] G. F. Chew et al.: Phys. Rev. 106 (1957) 1345

[29] T. W. Donnelly, A. S. Raskin: Ann. Phys. 169 (1986) 247

[30] D. Drechsel, L.Tiator: Journ. Phys. G 18, (1992) 449

[31] I. S. Barker, A. Donnachie, J. K. Storrow: Nucl. Phys. B 95, (1975) 347

[32] C. G. Fasano, F. Tabakin, B. Saghai: Phys. Rev. C 46 (1992) 2430

[33] E. Amaldi, S. Fubini, G. Furlan: Pion Electroproduction. Berlin: Springer 1979

[34] A. Donnachie, A. Shaw: Electromagnetic Interactions of Hadrons. New York: Plenum 1978

[35] S. Capstick, B. D. Keister: $\pi N$ Newsletter 8 (1993) 156;

S. Capstick, B. D. Keister: Phys. Rev. D 51 (1995) 3598

[36] Particle Data Group: Rev. Mod. Phys. 48 II (1976) 157

[37] L. A. Copley, G. Karl, E. Obryk: Nucl. Phys. B 14 (1969) 302

[38] M. Warns et al.: Z. Phys. C 45 (1990) 613; M. Warns et al.: Z. Phys. C 45 (1990) 627;

H. Schröder: Diploma Thesis (Bonn University, 1985)

[39] W. Konen, H. J. Weber: Phys. Rev. D 41 (1990) 2201

[40] D. M. Manley et al.: Phys. Rev. D 30 (1984) 904

[41] D. M. Manley, E. M. Saleski: Phys. Rev. D 45 (1992) 4002

[42] A. Donnachie: High Energy Physics V. Ed. E. H. S. Burhop. New York: Academic Press 1972) 
[43] J. M. Blatt, V. F. Weisskopf: Theoretical Nuclear Physics. New York: Wiley 1952)

[44] W. Grein, P. Kroll: Nucl. Phys. A 338 (1980) 332

[45] J. Piekarewicz: Phys. Rev. C 48 (1993) 1555

[46] J. M. Laget: Nucl. Phys. A 481 (1988) 765

[47] R. Brockmann, R. Machleidt: Phys. Rev. C 42 (1990) 1965

[48] K. M. Watson: Phys. Rev 95 (1954) 228

[49] B. Krusche: Proc. of II TAPS Workshop. Eds. J. Diaz, G. Martinez and Y. Schutz. Singapore: World Scientific 1994, 310; private communication 
Figure 1: Kinematics of an electroproduction experiment.

Figure 2: Frames for polarization vectors.

Figure 3: Electromagnetic helicity couplings of the resonance $S_{11}(1535)$ as function of momentum transfer. The solid line is the calculation with model M1 [37], the dotted line is the result of model M2 38], the dashed line the calculation with model M3 [39] and the dash-dotted line with model M4 [35].

Figure 4: Electromagnetic helicity couplings of the resonance $D_{13}(1520)$ as function of momentum transfer. For notation see Fig. 国.

Figure 5: Electromagnetic helicity couplings of the resonance $P_{11}(1440)$ as function of momentum transfer. For notation see Fig. E.

Figure 6: Total cross section for photoproduction. The left figure shows the inclusive cross section for eta photoproduction off the proton. The TAPS data [9] are denoted by $\square$, the Bonn data (०) are from an electroproduction experiment [12] at very low momentum transfer, $Q^{2}=0.056 \mathrm{GeV}^{2}$. In the energy regime beyond the resonance we have included some old data $(\diamond$ from [-4], * from [3]). The solid line is the full calculation with resonances and background, the dashed line is the calculation without background, the dotted line is the calculation without $D_{13}(1520)$ and $P_{11}(1440)$ resonance and the dash-dotted line is only the contribution of the non-resonant background. The right figure shows the same calculation for a neutron target.

Figure 7: Angular distribution for eta production off the proton at $\nu=783 \mathrm{MeV}$, compared to the data of the TAPS collaboration [9]. The solid line is the standard calculation, the long dashed line without Roper and the short dashed line without $D_{13}$ resonance. The dotted line was calculated with $\Gamma_{\eta} / \Gamma=0.003$ and the dash-dotted line with $\Gamma_{\eta} / \Gamma=0.010$ for the $D_{13}$.

Figure 8: Differential cross section for eta production off the proton (left) and the neutron (right) as a function of excitation energy $\nu$ in the lab frame and scattering angle $\Theta$ in the c.m. system.

Figure 9: Excitation functions off the proton for the unpolarized cross section $\sigma_{0}$, the single polarization observables and the double polarization observables for polarized beam and target at a scattering angle $\Theta=90^{\circ}$ in the c.m. system. The solid line is our standard calculation, the dotted line is without Roper resonance, the long dashed line without $D_{13}(1520)$, the short dashed line without $D_{15}(1675)$ and the dash-dotted line without non-resonant background. The data for $\sigma_{0}$ are from [9] (filled circles), [1] (boxes, $\Theta=95.7^{\circ}$ ), [6] (circles). The recoil polarization data are from [5].

Figure 10: Transverse/longitudinal separation of the inclusive cross section. The left figure shows the longitudinal part $\sigma_{L}$ of the inclusive electroproduction cross section on the proton. The data point is from [11. The dotted curve is the result of a calculation in the non-relativistic constituent quark model (M1) 37, 14. In the calculation for the short dashed curve, the resonance part of the $L_{0+}$ multipole was calculated in the relativized constituent quark model (M2) 38], the dash-dotted curve is the calculation in the light cone model [39] and the long dashed line is the contribution of the non-resonant background. The standard calculation (solid line) has been performed in model M1 with the $L_{0+}$ multipole normalized to the data point. The right figure shows the longitudinal cross section, $\varepsilon_{L} \sigma_{L}$ (dotted curve), and the transverse cross section $\sigma_{T}$ (dashed curve) in the standard calculation. The data point at $1533 \mathrm{MeV}$ is from [11].

Figure 11: Unpolarized differential cross sections $d \sigma_{T}^{00} / d \Omega$ and $d \sigma_{L}^{00} / d \Omega$ at $W=1533 \mathrm{MeV}$. The left figures are calculated at $Q^{2}=0.120 \mathrm{GeV}^{2}$, the right figures at $Q^{2}=0.393 \mathrm{GeV}^{2}$. The solid curve is our standard calculation (model M1), for the short dashed line the resonant parts of the $E_{0+}$ and $L_{0+}$ multipoles were calculated in model M3, the dotted line is a calculation without $P_{11}(1440)$, the long dashed line without $D_{13}(1520)$, and the dash-dotted line without non-resonant background. 
Figure 12: Unpolarized differential cross sections $d \sigma_{T T}^{00} / d \Omega$ and $d \sigma_{T L}^{00} / d \Omega$ at $W=1533 \mathrm{MeV}$. The left figures were calculated at $Q^{2}=0.120 \mathrm{GeV}^{2}$, the right figures at $Q^{2}=0.393 \mathrm{GeV}^{2}$. For notation see Fig. 国.

Figure 13: Differential cross sections $d \sigma_{T T}^{0 x} / d \Omega$ and $d \sigma_{T T}^{0 y} / d \Omega$ at $W=1533 \mathrm{MeV}$. The left figures were calculated at $Q^{2}=0.120 \mathrm{GeV}^{2}$, the right figures at $Q^{2}=0.393 \mathrm{GeV}^{2}$. For notation see Fig. E. 


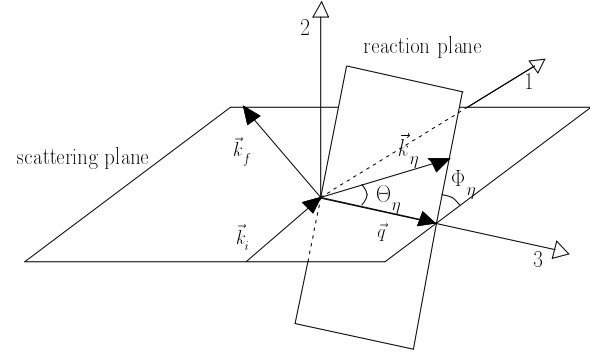




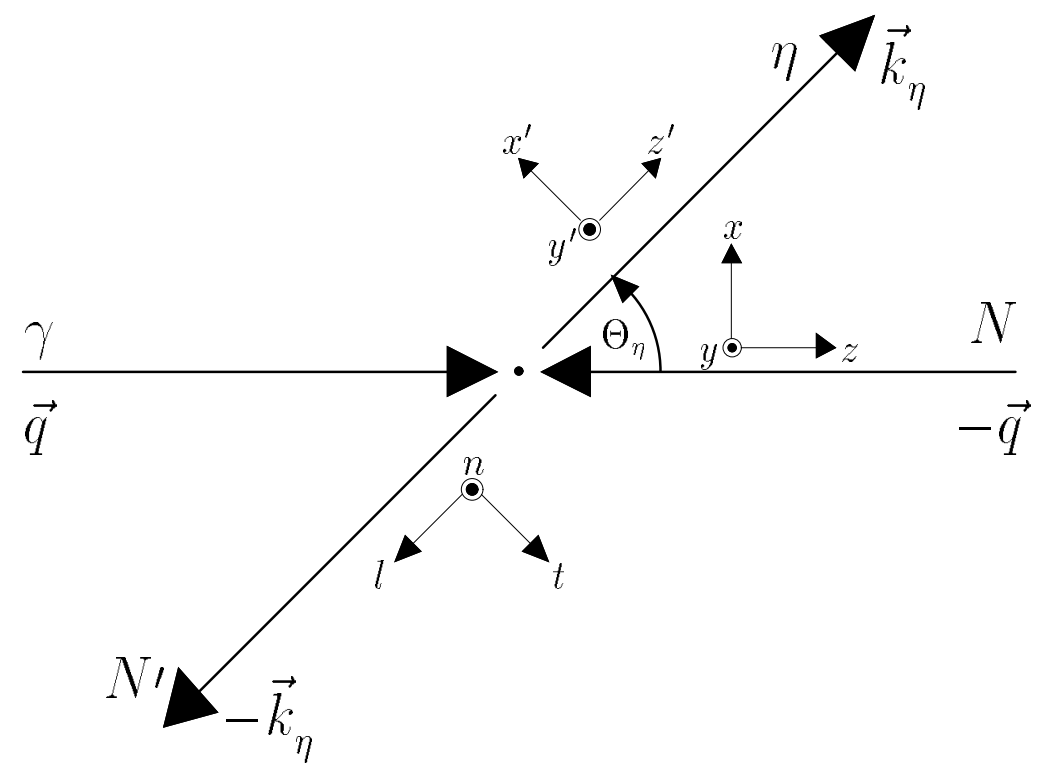



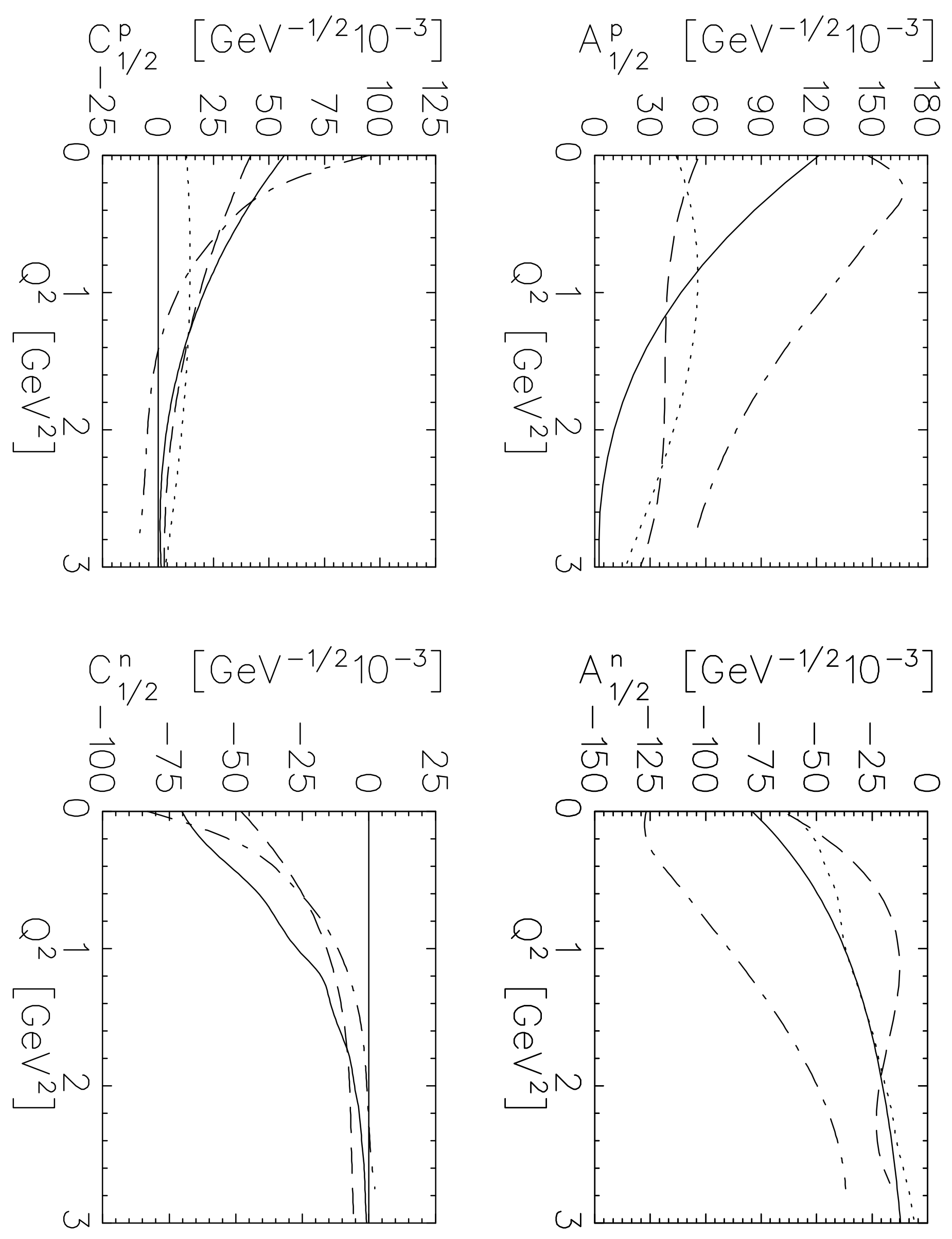

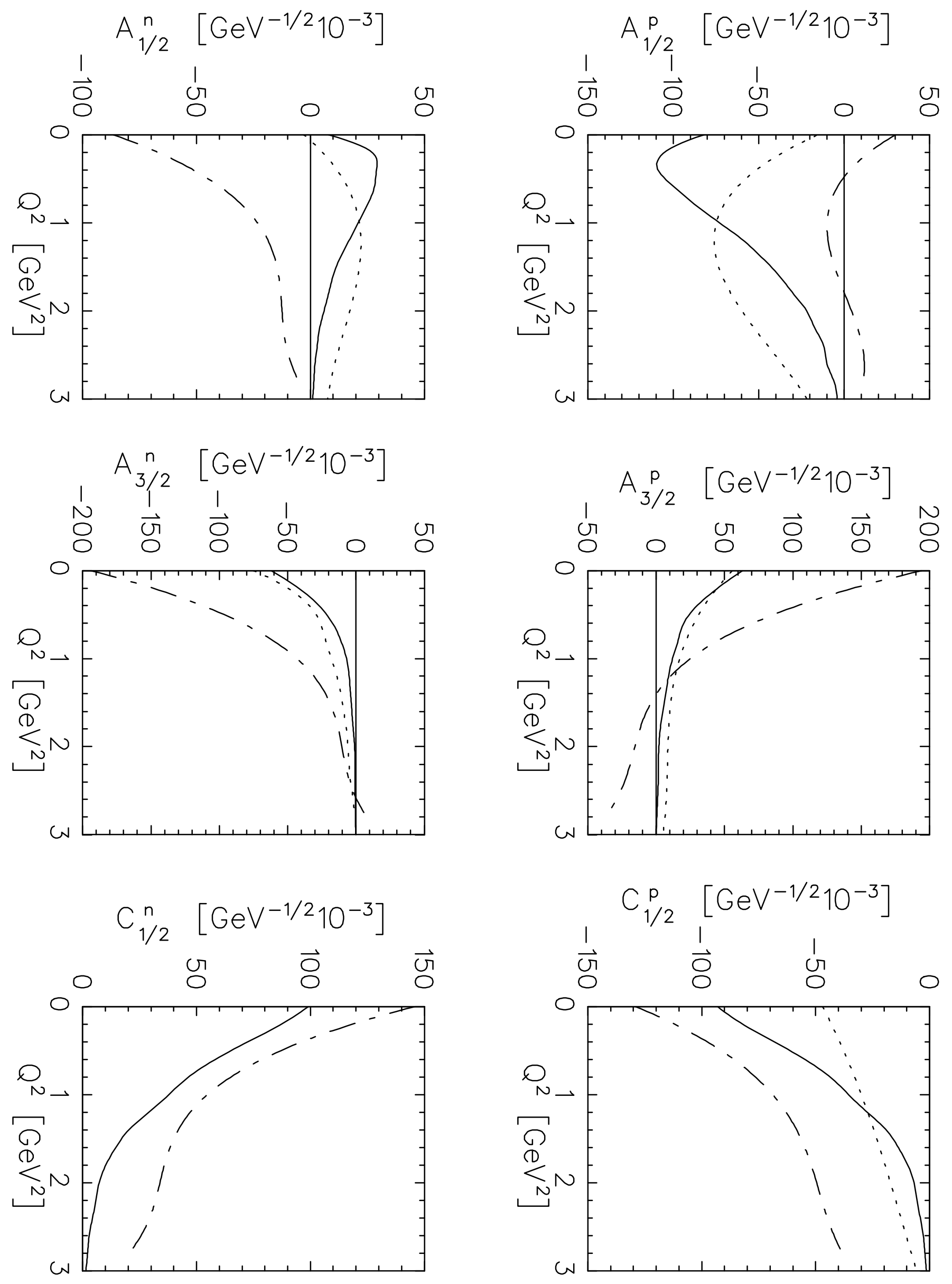

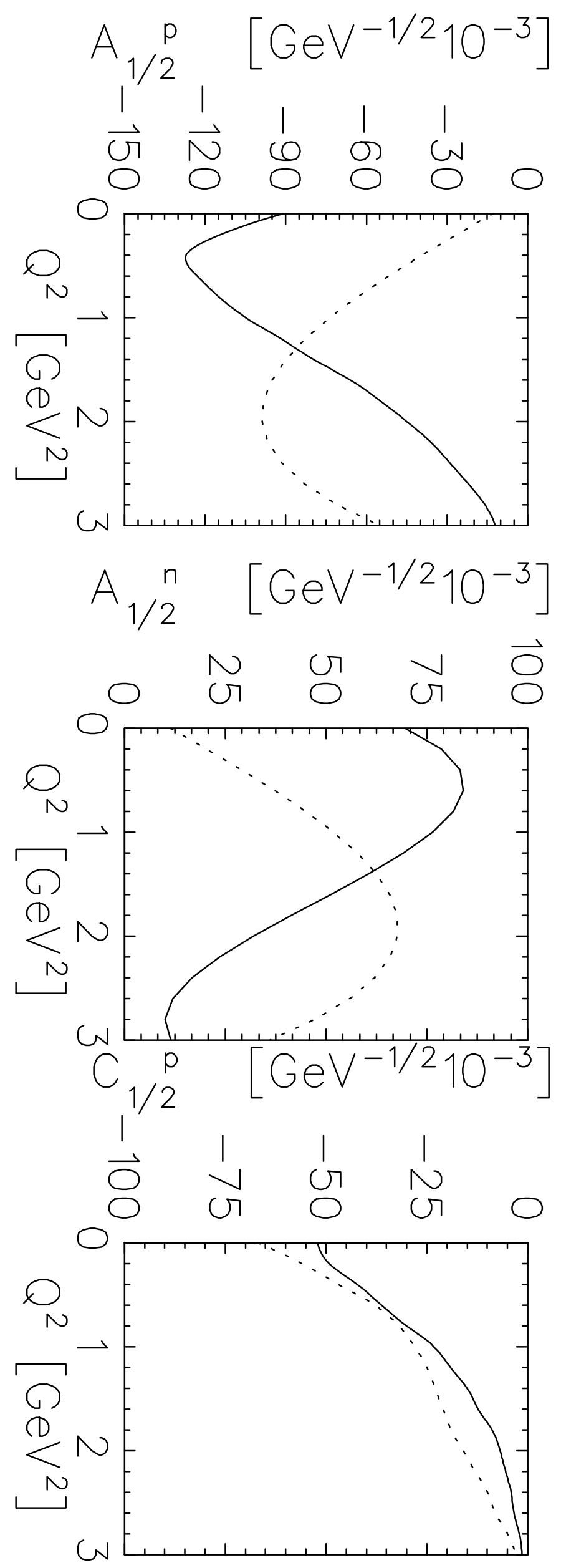

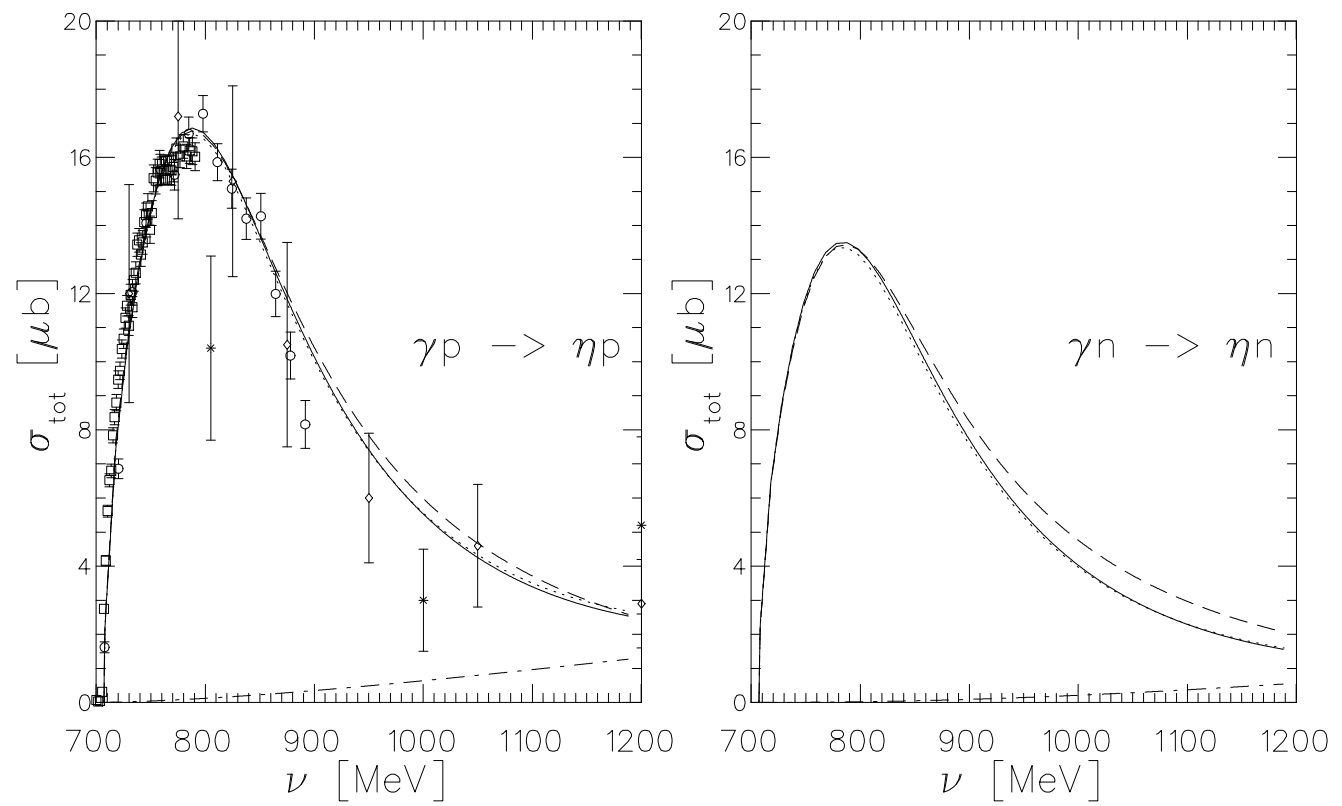


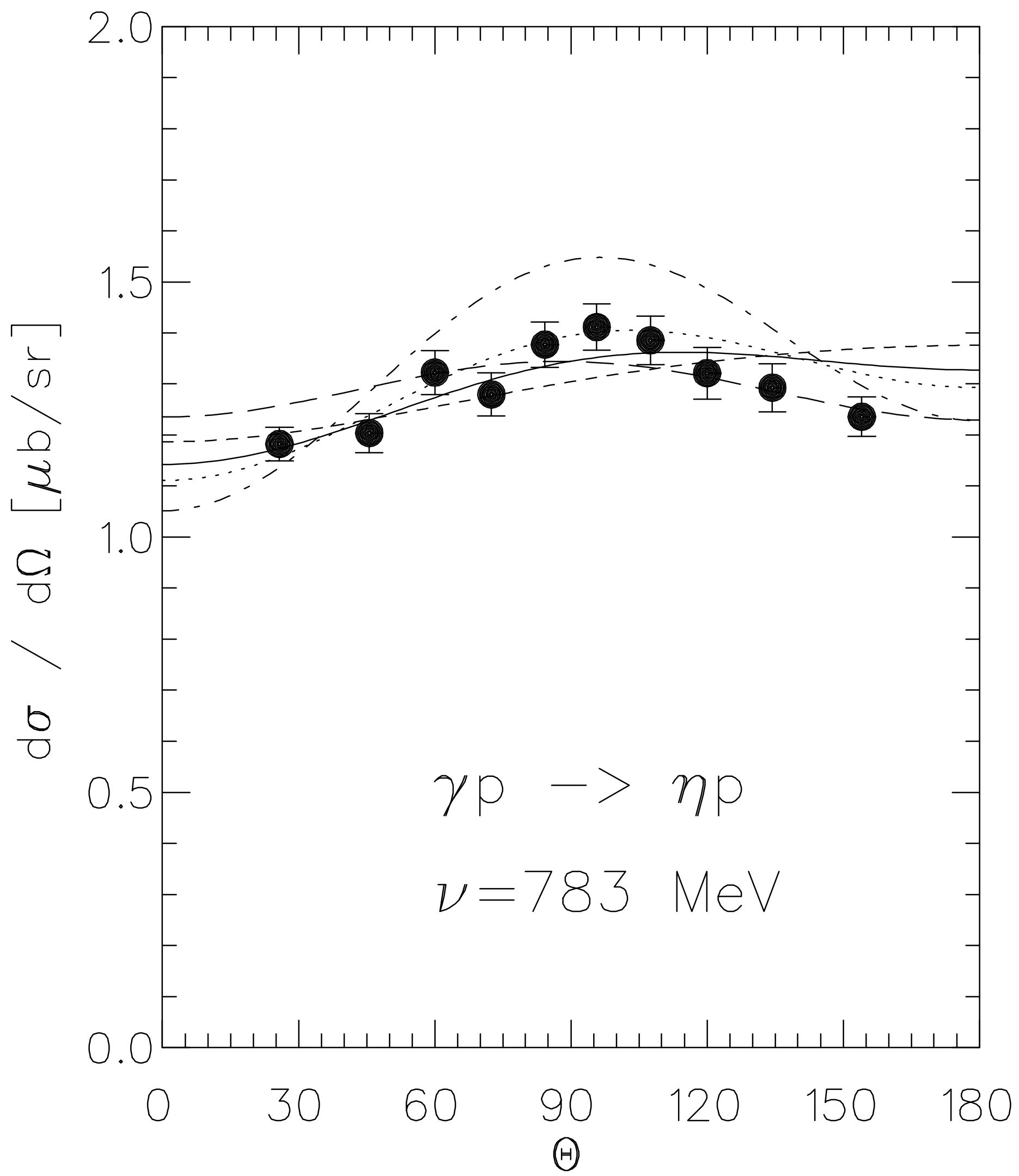



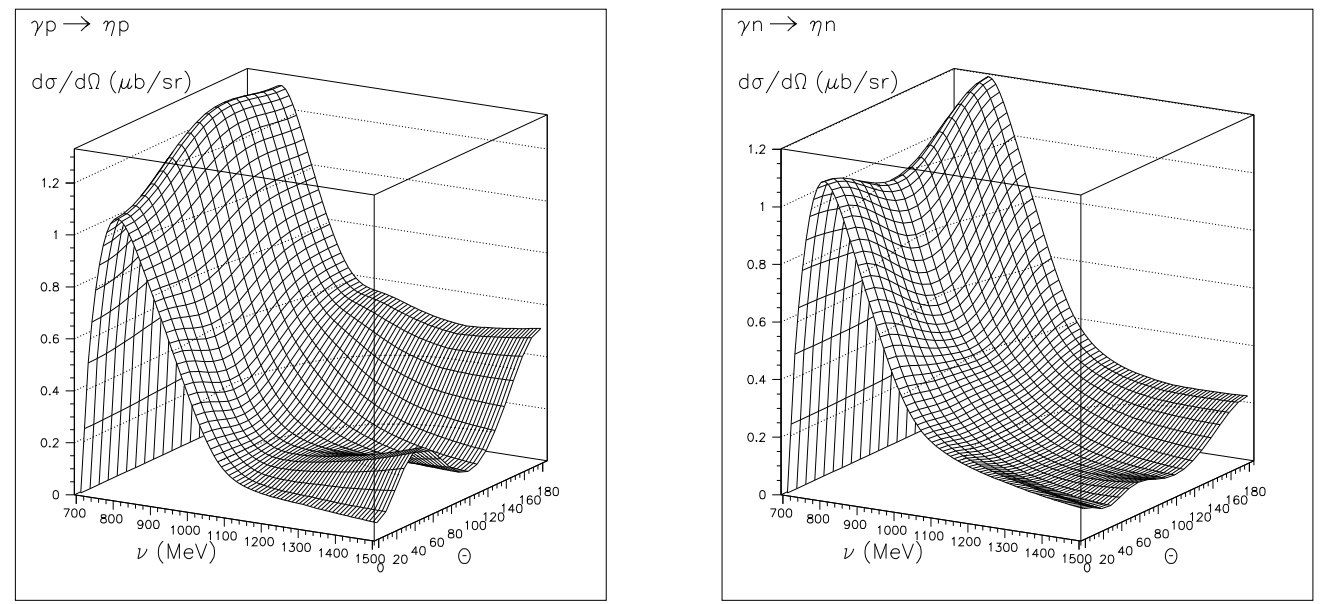

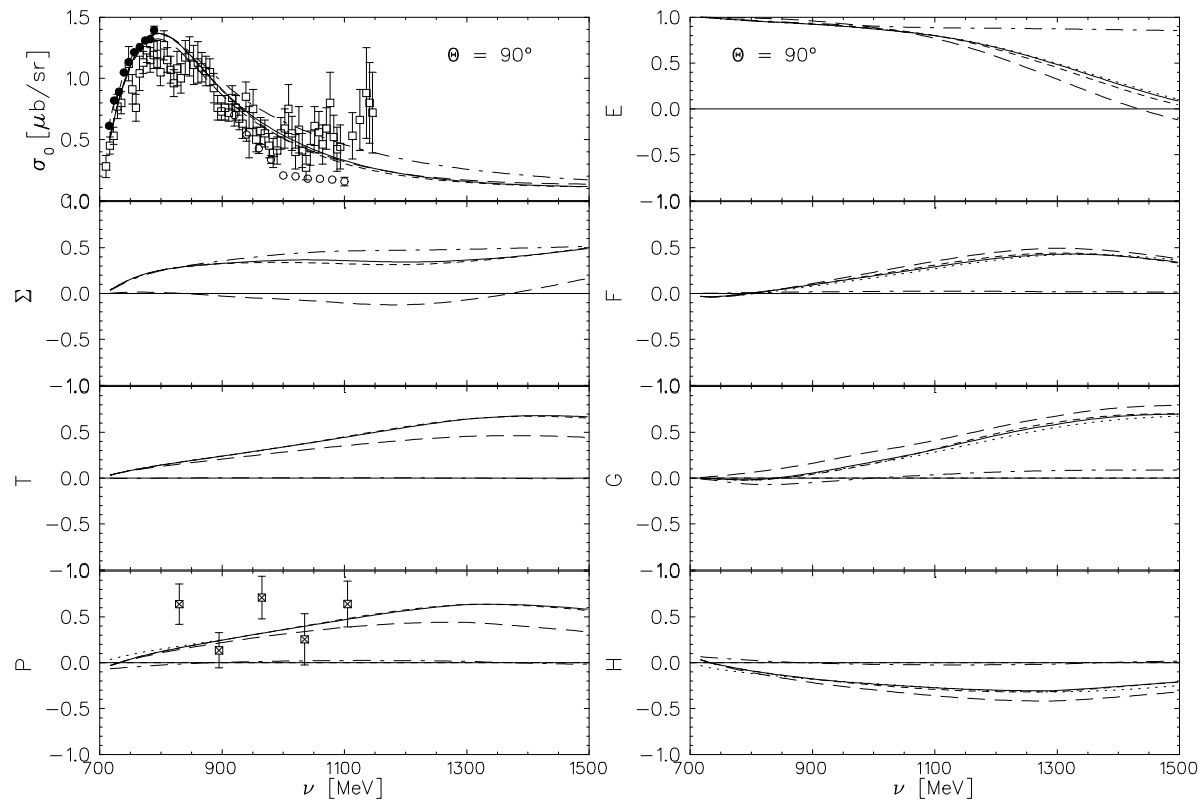

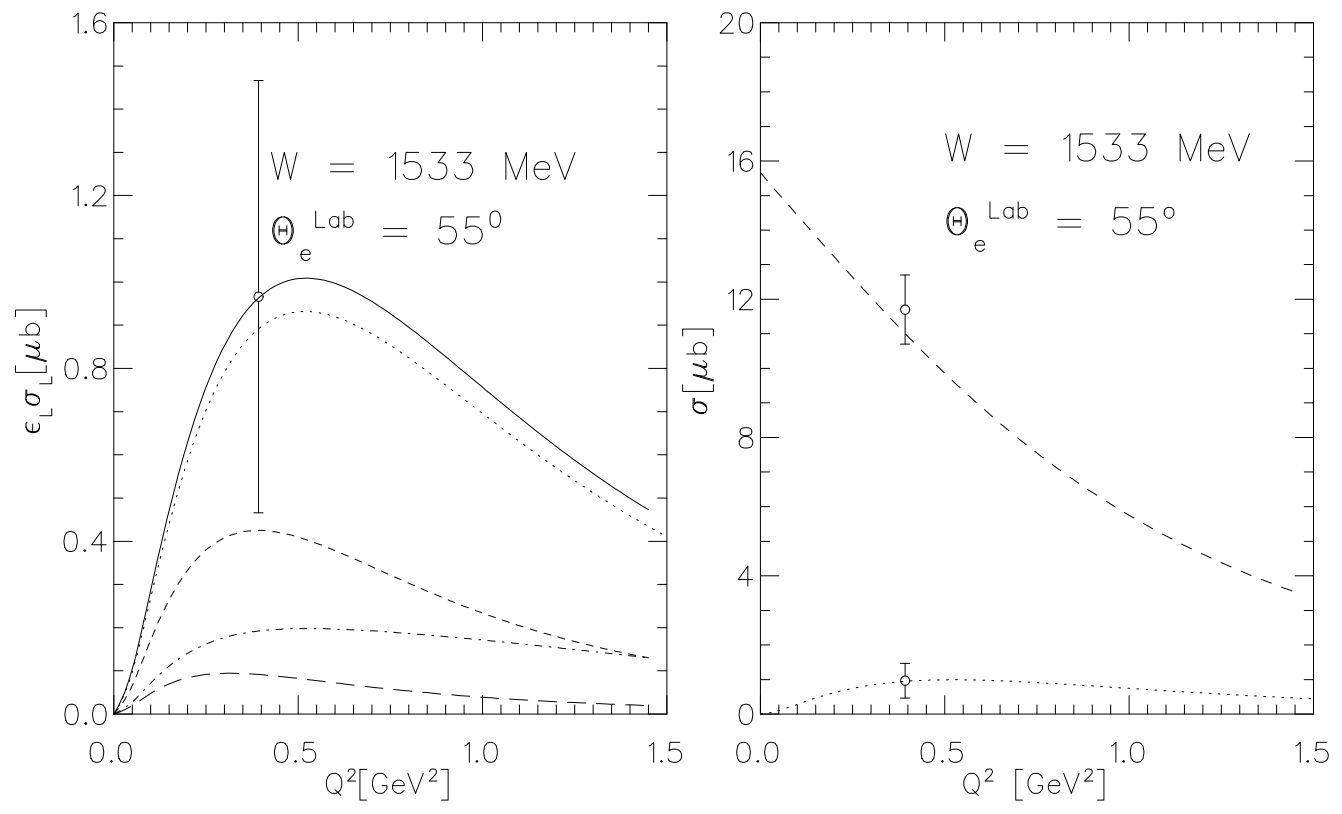

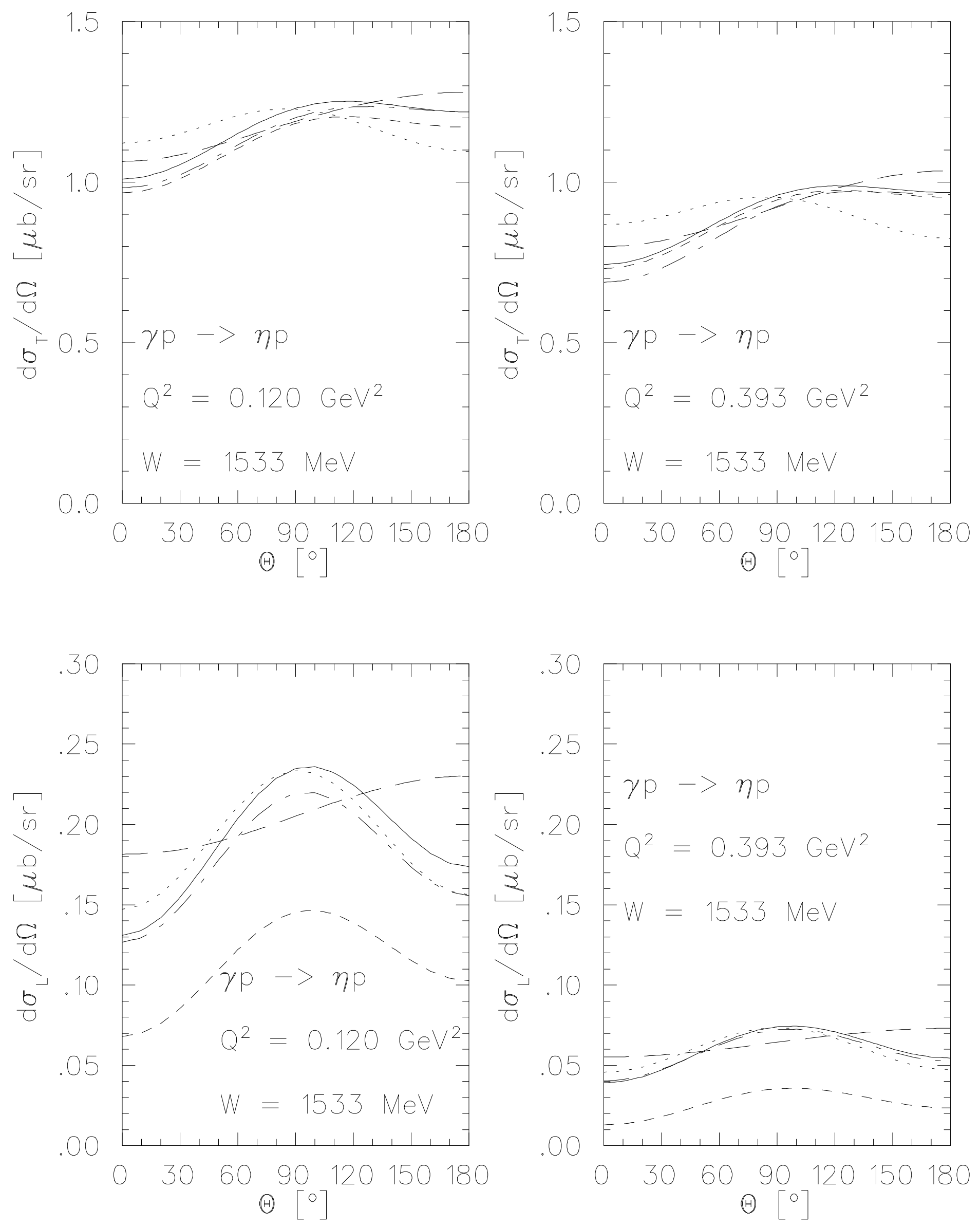

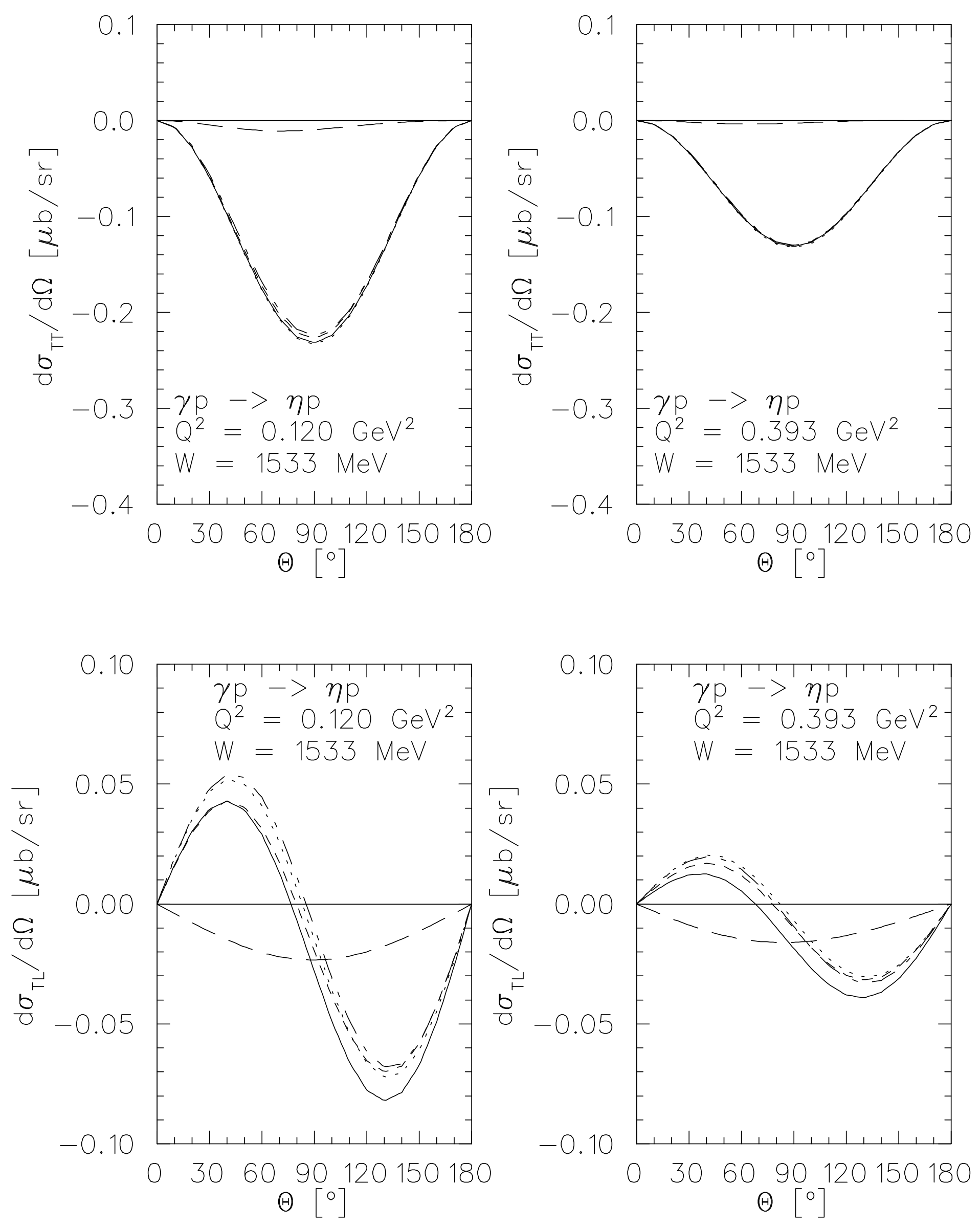

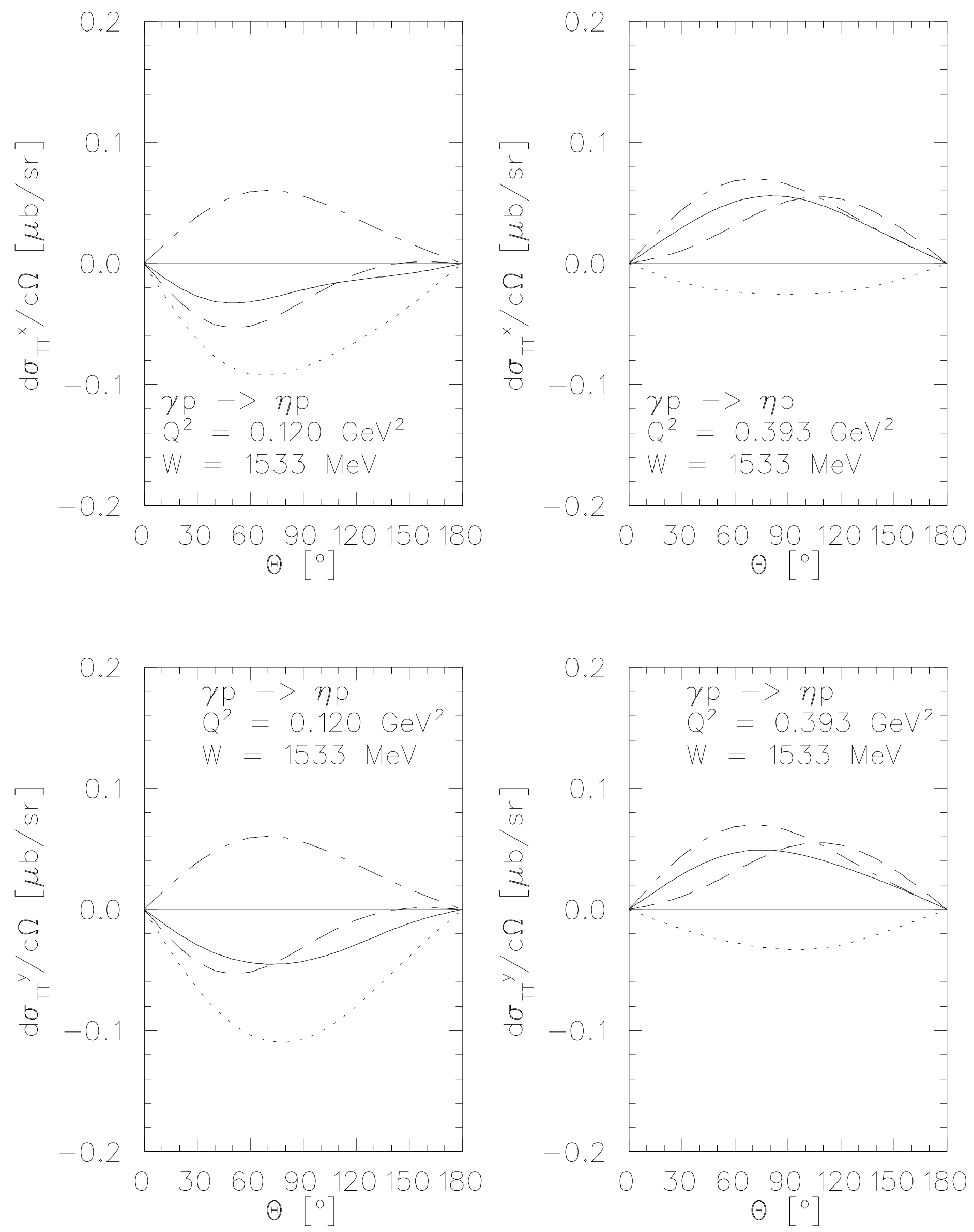